\title{
Dedicated Hippocampal Inhibitory Networks for Locomotion and Immobility
}

\author{
- Moises Arriaga and $\oplus^{-E d w a r d ~ B . ~ H a n ~}$ \\ Washington University School of Medicine, St. Louis, Missouri 63110
}

Network activity is strongly tied to animal movement; however, hippocampal circuits selectively engaged during locomotion or immobility remain poorly characterized. Here we examined whether distinct locomotor states are encoded differentially in genetically defined classes of hippocampal interneurons. To characterize the relationship between interneuron activity and movement, we used in vivo, two-photon calcium imaging in CA1 of male and female mice, as animals performed a virtual-reality (VR) track running task. We found that activity in most somatostatin-expressing and parvalbumin-expressing interneurons positively correlated with locomotion. Surprisingly, nearly one in five somatostatin or one in seven parvalbumin interneurons were inhibited during locomotion and activated during periods of immobility. Anatomically, the somata of somatostatin immobility-activated neurons were smaller than those of movementactivated neurons. Furthermore, immobility-activated interneurons were distributed across cell layers, with somatostatin-expressing cells predominantly in stratum oriens and parvalbumin-expressing cells mostly in stratum pyramidale. Importantly, each cell's correlation between activity and movement was stable both over time and across VR environments. Our findings suggest that hippocampal interneuronal microcircuits are preferentially active during either movement or immobility periods. These inhibitory networks may regulate information flow in "labeled lines" within the hippocampus to process information during distinct behavioral states.

Key words: behavior; calcium imaging; circuits; hippocampus; interneurons; virtual reality

Significance Statement

The hippocampus is required for learning and memory. Movement controls network activity in the hippocampus but it's unclear how hippocampal neurons encode movement state. We investigated neural circuits active during locomotion and immobility and found interneurons were selectively active during movement or stopped periods, but not both. Each cell's response to locomotion was consistent across time and environments, suggesting there are separate dedicated circuits for processing information during locomotion and immobility. Understanding how the hippocampus switches between different network configurations may lead to therapeutic approaches to hippocampal-dependent dysfunctions, such as Alzheimer's disease or cognitive decline.

\section{Introduction}

Neuronal networks compute information in a state-dependent manner, in which current behavior alters circuit computations. This has been clearly demonstrated in Caenorhabditis elegans and in the stomatogastric ganglion of crabs, where neuronal circuits have dramatically different output patterns, depending on the

Received April 18, 2017; revised Aug. 8, 2017; accepted Aug. 13, 2017.

Author contributions: M.A. and E.B.H. designed research; M.A. and E.B.H. performed research; M.A. analyzed data; E.B.H. wrote the paper.

This work was supported by the McDonnell Centers for Systems Neuroscience and Cellular and Molecular Neurobiology. We thank Martha Bagnall, Azad Bonni, Tim Holy, Andreas Burkhalter, Mike Nonet, David van Essen, Michael Bruchas, and Larry Snyder for comments and discussion; Andreas Burkhalter and Jin-Moo Lee for mutant mice; and Akshata Korgaonkar and Elena Waidmann for sectioning and immunostaining. GCaMP and jRGECO were provided courtesy of the GENIE (Genetically-Encoded Neuronal Indicator and Effector) project (Janelia Research Campus).

The authors declare no competing financial interests.

Correspondence should be addressed to Edward B. Han, Washington University School of Medicine, Department of Neuroscience, 660 South Euclid Avenue, Campus Box 8108, St. Louis, M0 63110. E-mail: han@pcg.wustl.edu.

DOI:10.1523/JNEUROSCI.1076-17.2017

Copyright $\odot 2017$ the authors $\quad 0270-6474 / 17 / 379222-17 \$ 15.00 / 0$ state of the animal (Bargmann and Marder, 2013). Movement and immobility are two locomotion states that have wide-ranging effects across rodent sensory systems, increasing stimulus gain in the visual cortex (Niell and Stryker, 2010; Polack et al., 2013; Reimer et al., 2014; Vinck et al., 2015), while decreasing sensory responses in the auditory cortex (Schneider et al., 2014; McGinley et al., 2015). The differential effects of movement and immobility extend into the hippocampus, where they are associated with two strikingly distinct forms of network activation (Vanderwolf, 1969). During movement, local field potential (LFP) activity is characterized by $\theta$-frequency oscillations $(7-12 \mathrm{~Hz})$, and pyramidal neurons in CA3 and CA1 exhibit place-specific firing (O'Keefe and Dostrovsky, 1971; McNaughton et al., 1983; Wilson and McNaughton, 1993). In contrast, during awake immobility, LFP activity is punctuated by large, irregular activity containing periods of sharp-wave/ripple (SWR) events, during which pyramidal neurons fire in compressed temporal sequences that can replay recently experienced trajectories (Wilson and McNaughton, 1994; 
Foster and Wilson, 2006; Karlsson and Frank, 2009; Buzsáki, 2015; Colgin, 2016).

Not only is movement important for controlling hippocampal network state but an internal representation of speed is a critical parameter for path integration and spatial navigation. Indeed, movement speed positively modulates firing in pyramidal neurons and in many classes of interneurons in the hippocampus, as well as in the strongly interconnected medial septum (MS) and entorhinal cortex (McNaughton et al., 1983; Ahmed and Mehta, 2012; Varga et al., 2012; Katona et al., 2014; Fuhrmann et al., 2015; Kropff et al., 2015; Zheng et al., 2015; Hinman et al., 2016). Neurons engaged by the opposite motor state, immobility, are far less studied. Recently, researchers identified a subset of hippocampal pyramidal neurons that is preferentially active during periods of immobility and encodes the animal's current location (Kay et al., 2016). However, little is known about interneurons that control activity during these periods of immobility. This is a critical question given the powerful role of inhibition in controlling network state (Ellender et al., 2010) and downstream behaviors. Recent studies have revealed that different subsets of interneurons are required for diverse behaviors, including fear learning (Letzkus et al., 2011; Lovett-Barron et al., 2014), sensorimotor integration (Gentet et al., 2012; Lee et al., 2013), visual system gain (Fu et al., 2014; Pakan et al., 2016), and memory formation and expression (Courtin et al., 2014; Morrison et al., 2016; Stefanelli et al., 2016).

Intriguingly, several investigators have reported putative interneurons activated by immobility (Fox and Ranck, 1975; Buzsáki et al., 1983; Colom and Bland, 1987; Mizumori et al., 1990; Csicsvari et al., 1999). However, the rarity of neurons that show this property (1.6\% of all hippocampal neurons by one study; Mizumori et al., 1990), coupled with the limited information about cellular properties and anatomical localization obtained by extracellular recording, has left this population poorly described. To overcome these limitations, we used two-photon calcium imaging in awake, behaving mice. By using cre-driver lines to target our recordings to specific subtypes of interneurons (Taniguchi et al., 2011), we functionally characterized hundreds of interneurons during behavior and collected detailed anatomical information on their locations.

We found that hippocampal neurons specialize in distinct locomotion states. In the majority, activity was positively correlated with locomotion, but a distinct subset was activated specifically by immobility. These functionally defined interneurons were present in both somatostatin-expressing and parvalbuminexpressing inhibitory interneurons and were anatomically distributed across stratum oriens ( $\mathrm{SO}$ ) and stratum pyramidale (SP). Finally, each neuron's activity correlation to movement was stable both across time and virtual-reality (VR) environments, suggesting that this cellular characteristic represents a static, rather than dynamic, function in the hippocampal network.

\section{Materials and Methods}

Animals. All experiments were approved by the Washington University Animal Care and Use Committee. Hetereozygotes $(+/-)$ from two credriver mice lines on a $\mathrm{C} 57 \mathrm{Bl} / 6 \mathrm{~J}$ genetic background were used to label parvalbumin-expressing and somatostatin-expressing inhibitory in-

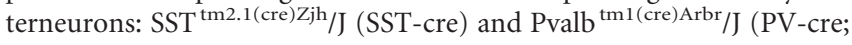
Jackson Labs). Wild-type (WT) mice (C57BL/6J) were cre-negative pups from the SST-cre ${ }^{+/-} \times$WT crossing (littermate controls). Both male and female mice were used.

Viral injections and hippocampal window implantation. Mice were injected with adeno-associated virus (AAV) at 2-4 months of age. Mice were anesthetized with $1-3 \%$ isofluorane and a $0.5-\mathrm{mm}$-diameter crani- otomy was opened above the left cortex. Injections were made through a micropipette tip pulled to a long, thin taper and sharpened on a Sutter Instruments BV-10 beveler to an input impedance of $2-5 \mathrm{M} \Omega$. Virus was pressure-injected and volume ( $\sim 50 \mathrm{nl})$ was estimated by visually measuring the movement of the back meniscus of virus in the pipette using an eyepiece reticule. For stereotactic injections, virus was targeted to the CA1 layer of the hippocampus at -1.6 to $-1.8 \mathrm{~mm}$ lateral from bregma, -1.7 to $-2.0 \mathrm{~mm}$ caudal from bregma, and -1.3 to $1.35 \mathrm{~mm}$ ventral from dura. For credependent GCaMP6f expression, AAV1.Syn.Flex.GCaMP6f.WPRE.SV40 (Penn Vector Core, University of Pennsylvania) at a titer of $1.71 \times 10^{13}$ genome copies (g.c.) was diluted 1:1-1:4 with PBS and injected in cre-driver mice. For neuronal jRGECO1a expression, AAV1.Syn.NES-jRGECO1a. WPRE.SV40 (Penn Vector Core, University of Pennsylvania) at a titer of $2.95 \times 10^{13}$ g.c. was diluted 1:1 with PBS and injected into WT mice. In two somatostatin-cre mice, we made bilateral injections $(300 \mathrm{nl})$ of a mixture of AAV1.Syn.Flex.GCaMP6f.WPRE.SV40 at a 1:5 dilution with rAAV8/hSyn-DIO-hm3D (Gq)-mcherry (UNC Vector Core, University of North Carolina; $5.7 \times 10^{12}$ g.c.) at a 4:5 dilution to coexpress GCaMP6f and Gq-DREADD (designer receptors exclusively activated by designer drugs) in cre-expressing neurons. We did not activate DREADD receptors with the exogenous ligand $\mathrm{CNO}$ in any experiments presented here. GCaMP6f signaling in these mice was similar to that of mice expressing GCaMP6f only, so data from both types of animals were combined.

After virus injection, the incision was sealed with Metabond (Parkell) and a custom-cut titanium headplate (eMachineShop). Mice were waterscheduled to bring weight down to $\sim 75 \%$ of original $(0.7-1 \mathrm{ml}$ of water per day). After 1-3 weeks, the headplate was removed, a larger craniotomy $(2.8 \mathrm{~mm})$ was made, the cortex overlying the hippocampus was aspirated, and the top layers of the external capsule were removed, leaving the lower layers intact. This aspiration unilaterally removes parts of the visual, somatosensory, and parietal cortices. Previous studies found this surgery did not impair mouse behavior in numerous tasks, including VR track running (Dombeck et al., 2010; Gu et al., 2014). Anecdotally we also did not observe an obvious deficit when comparing the VR task behavior of mice implanted with an imaging cannula to mice that were only headplated.

Kwik-Sil elastomer (World Precision Instruments) was used to bond the imaging cannula [outer diameter, $2.8 \mathrm{~mm}$; inner diameter, $2.36 \mathrm{~mm}$; height, $1.5 \mathrm{~mm}$ (Microgroup); $2.5 \mathrm{~mm}$ round coverslip (Potomac Photonics)] to the brain and Metabond darkened with carbon powder (Sigma-Aldrich) to prevent VR light from entering the objective was used to close the incision and attach the headplate. Cannulae were inserted at a slight angle $\left(\sim 7-10^{\circ}\right.$ down toward the lateral side) to match the angle of the lateral side of the hippocampus. During imaging, the objective was tilted a similar amount so the long axis of the objective was perpendicular to the imaging window. Animals recovered for $\geq 2$ weeks with continued water-scheduling before beginning experiments. Animals were imaged 1-3 months after virus injection. We observed no signs of toxic overfilling of neurons (filled nuclei and long-time course transients) by calcium sensor over this time frame.

VR track running behavior. The VR system used a curved-screen monitor (Samsung S34E790C) set on lowest brightness and further dimmed using window film (Gila Glare Control, Smoke). The monitor was $\sim 12$ inches in front of the mouse and occupied $115^{\circ}$ of horizontal (azimuth) space and $-15^{\circ}$ below to $+39^{\circ}$ above the horizon of the mouse. The mouse was head-fixed on a spherical treadmill (8-inch-diameter Styrofoam ball) floating on a column of air flowing through a custom designed, 3D-printed treadmill base. Mouse movement was tracked by monitoring ball movement using a G400 mouse (Logitech) configured in LabView (National Instruments) to read forward and yaw (rotation) ball speed. ViRMEn (Virtual Reality Matlab Engine; Aronov and Tank, 2014) was used to render a closed-loop visual VR environment based on movement input from the computer mouse. The forward ball movement gain was set so that $\sim 2.8$ rotations of the ball (equal to $180 \mathrm{~cm}$ of distance traveled) traversed the long axis of the track. Yaw gain was set so $\sim 12$ ball rotations equaled a $360^{\circ}$ rotation in VR in the track while in the end zones (last $10 \mathrm{~cm}$ of the track on either end, i.e., $0-10$ and $170-180 \mathrm{~cm}$ ) gain was set so $\sim 2.5$ ball rotations equaled a $360^{\circ}$ rotation, which allows mice to more easily turn out of corners at the end of the track. Rewards were 
controlled by a transistor-transistor logic (TTL) output from the VR engine and were given at alternating ends of the VR track. Each reward consisted of two rewards of 3-4 $\mu$ l of water each, separated by a $500 \mathrm{~ms}$ interval. Animals were trained $\sim 21.5 \mathrm{~min}$ per day, $5-6 \mathrm{~d}$ per week, for 2-3 weeks until they could consistently performed at $>2.5$ rewards $/ \mathrm{min}$. Most imaging sessions were $21.5 \mathrm{~min}$. In remapping experiments, the visual environment was instantaneously switched to a new world. This switch occurred when the animal was in the track, not in a reward zone, and was marked by a single reward. Animals spent $7 \mathrm{~min}$ in World 1 and $14 \mathrm{~min}$ in World 2. Animals were familiarized with the remapping protocol and World 2 for $4-5 \mathrm{~d}$ (one session per day) before imaging during remapping.

Two-photon imaging. In vivo calcium imaging was done on a Neurolabware laser-scanning two-photon microscope equipped with an electric tunable lens (ETL; Optotune, EL-10-30-NIR-LD) in the excitation light path, in combination with a $\mathrm{f}=-100 \mathrm{~mm}$ offset lens, to rapidly modulate the axial focus by altering laser divergence (Grewe et al., 2011). The ETL is in the excitation light path before the galvos and does not impinge on light collection. We sequentially imaged multiple planes spanning distances of $\leq \sim 180 \mu \mathrm{m}$ in the $z$-axis. The focal plane was controlled by current output, which in turn was controlled by microscope software (Scanbox, Neurolabware). We typically imaged 1-6 axial planes, with most movies (22 of 31 ) at four planes. Total frame rate was $15.5 \mathrm{~Hz}$ for unidirectional line scanning or $31 \mathrm{~Hz}$ for bidirectional. Collecting four axial planes in unidirectional mode results in a frame rate of $3.9 \mathrm{~Hz}$ at each plane $(15.5 \mathrm{~Hz} / 4$ planes). Settling time of the ETL was typically $\sim 10 \mathrm{~ms}$, which can be observed as an oscillation in the focal depth over the first several lines of each plane of view. This area was cropped out for analysis. Emitted fluorescence light was detected by GaAsP photo-multiplier tubes (Hamamatsu, 10770-40) after bandpass filtering (Semrock, BrightLine 510/84 for GCaMP6f and 607/70 for jRGECO1a). Movies were collected through a $16 \times, 0.8$ numerical aperture objective (Nikon, CFI 75 LWD 16X) at $796 \times 512$ pixels corresponding to fields of view of $\sim 500 \times 500$ or $1000 \times 1000 \mu \mathrm{m}$ depending on galvo gain settings. Ti:sapphire laser (MaiTai, Spectra-Physics) light was tuned to $920 \mathrm{~nm}$ for GCaMP6f imaging and $1020 \mathrm{~nm}$ for jRGECOla imaging. Laser power was $\sim 25-50 \mathrm{~mW}$ after the objective and was set independently for each plane imaged using a pockels cell (ConOptics, 350-80-LA-02 KD, BK option, 302RM driver). Detailed $z$ series of imaged areas were taken with $3 \mu \mathrm{m} z$ steps and averaging of 10-40 frames per plane. Raw ball-speed data (forward and yaw, measured by computer mouse and exported via a National Instruments PCI-6221 data-acquisition board), VR data (track position, head direction, rewards, exported from the VR computer via a National Instruments PCI-6229 data-acquisition board), and imaging timing (each imaging frame was marked by a TTL output from the microscope-controller board) were synchronized by collecting all data in a single file using pClamp (RRID:SCR_011323) via a Digidata 1322 digitizer (Molecular Devices).

Immunofluorescence labeling. Naive WT adult mice were perfused with $4 \%$ paraformaldehyde in PBS, postfixed overnight, and cryoprotected in $30 \%$ sucrose. Coronal sections $(50 \mu \mathrm{m})$ were made on a freezing microtome. Sections were washed in $0.1 \mathrm{M}$ Tris-buffered saline (TBS) and blocked in $5 \%$ normal donkey serum, $3 \%$ bovine serum albumin (BSA) and $0.1 \%$ Triton in $0.5 \mathrm{~m}$ TBS for $2 \mathrm{~h}$. For labeling, sections were incubated overnight in anti-somatostatin 28 antibody (1:500; ImmunoStar, catalog \#20089, RRID:AB_572265) or anti-parvalbumin antibody (1:1000; Swant, catalog \#PVG-213, RRID:AB_2650496) in 1\% normal donkey serum, $2 \%$ BSA, $0.1 \%$ Triton in $0.5 \mathrm{M}$ TBS PBS at $4{ }^{\circ} \mathrm{C}$. Sections were washed in $0.1 \mathrm{M}$ TBS and reacted with fluorescent secondary antibodies for $2 \mathrm{~h}$ at room temperature: donkey anti-rabbit Cy5 (1:500; Jackson ImmunoResearch Labs, catalog \#711-175-152, RRID:AB_2340607) or donkey anti-Goat Cy5 (1:500; Jackson ImmunoResearch Labs, catalog \#705-175-147, RRID:AB_2340415). Sections were mounted on slides using Vectashield (Vector Laboratories, catalog \#H-1000, RRID:AB_2336789) and imaged on a Nikon Eclipse 90i microscope with a Cool Snap HQ CCD camera (Photometrics) controlled by Volocity software (PerkinElmer). Quantification was performed in SO and SP of CA1. Cells were counted manually from every sixth section of the hippocampus $(2-4$ sections per mouse).
Data analysis. Data were analyzed using custom programs written in Matlab (RRID:SCR_001622). Images were motion-corrected using cross-correlation registration and rigid translation of individual frames (Dombeck et al., 2010). Slow fluctuations in fluorescence were removed by taking a window $\sim 15 \mathrm{~s}$ around each time point and subtracting the eighth percentile of fluorescence intensity distribution within that window. For jRGECO labeling of all neurons, putative interneurons were eliminated by excluding cells outside of SP and eliminating cells with $\Delta F / F$ skew $<5$. ROIs were defined using principal component analysis/ independent component analysis as described previously (Mukamel et al., 2009; Dombeck et al., 2010) with an additional step or removing ROIs that spatially overlapped by $>20 \%$. To identify pyramidal neurons identified in multiple $z$ planes, pairs of cells with temporal activity correlation $>0.7$ were flagged and visually inspected. The dimmer ROI was removed when duplicates were identified.

For interneuron imaging, ROIs were selected in a semiautomated process. Possible ROIs were automatically identified as contiguous regions with SD $>1.5$ and an area $>90 \mu \mathrm{m}^{2}$. ROIs that were unresponsive or had low signal-to-noise ratios were manually eliminated. The parameters for automatic detection were permissive so many puncta were identified that were clearly not cells. This was especially common in the upper imaging planes, where there is fluorescent background near the surface of the hippocampus (Fig. 1B, somatostatin interneurons, plane 1, upper right corner). Puncta that were unresponsive or had low signal-to-noise ratios were dropped from further analysis ( 65 of 286 for somatostatin interneurons and 109 of 234 for parvalbumin interneurons). In movies with multiple planes, individual cells were inspected in multiple planes so that the same cell was not selected multiple times and the ROI was selected on the plane where the cell was most sharply in focus. For interneuron recordings, neuropil contamination was removed by subtracting perisomatic fluorescence signal from an area $<20 \mu \mathrm{m}$ from the ROI border, excluding any other ROIs ( $\left.F_{\text {Corrected-ROI }}=F_{\text {ROI }}-F_{\text {Neuropil }}\right)$; Peron et al., 2015).

Ball-movement data, collected at $1 \mathrm{kHz}$, was binned to match imaging frame rate. Ball speed was calculated from the voltage output of the LabView program that detects ball movement. To generate a conversion factor for voltage output to ball speed, we manually spun the ball at different speeds to traverse the VR track. Since the VR track length was set to be the equivalent of $180 \mathrm{~cm}$ of ball distance, we could use travel in VR as a measure of real-world distance. We calculated distance over time as ball speed and generated a lookup table of ball speed versus voltage output. This function was linear for speeds that approximate mouse movement speeds. The same calibration was made for yaw ball speed. Total ball speed was calculated as the magnitude of the Euclidean $x$ and $y$ components of velocity (forward and yaw rotation) as sampled by the computer mouse. Acceleration was calculated as the difference between subsequent values of the calculated ball speed.

Activity fields for neurons were calculated as full width of the field (in centimeters) at half-maximal $\Delta F / F$ of peak activity. Spatial information was calculated with $5 \mathrm{~cm}$ bins using the following formula: SI $=\Sigma P_{i}\left(R_{i} / R\right) \log _{2} R_{i} / R$ (Markus et al., 1994) where $P_{i}$ is the probability of bin occupancy, $R_{i}$ is the mean $\Delta F / F$ in bin $i$, and $R$ is overall mean $\Delta F / F$. The locomotion modulation index (LMI) was calculated for individual cells as the difference between the mean fluorescence during immobile and moving periods, normalized by the sum of the mean values, $L M I=\frac{\mu_{m}-\mu_{s}}{\mu_{m}+\mu_{s}}$. Activity $(\Delta F / F)$ is displayed versus linear ball speed. Previous reports have shown activity having a linear relationship to log speed (Ahmed and Mehta, 2012; Kemere et al., 2013; Zheng et al., 2015), as well as linear speed (Kropff et al., 2015; Hinman et al., 2016). In general, activity was better fit to linear speed for somatostatin neurons, so we used linear speed throughout (122 of 192 or $59.9 \%$ better fit by linear ball speed, as measured by the sum of square residuals to the linear regression after excluding the highest $20 \%$ of speed-activity values because of activity saturation). Interestingly, there appear to be cell-type differences in the relationship between activity and speed, since parvalbumin interneurons, on the whole, were better fit to $\log _{2}$ ball speed ( 76 of 125 or $60.8 \%$ better fit to $\log _{2}$ ). For morphometric analysis, ROIs were redrawn by hand over the cell soma, blind to the cell's activity correlation with speed. Cross-sectional somatic area and eccentricity were calculated 


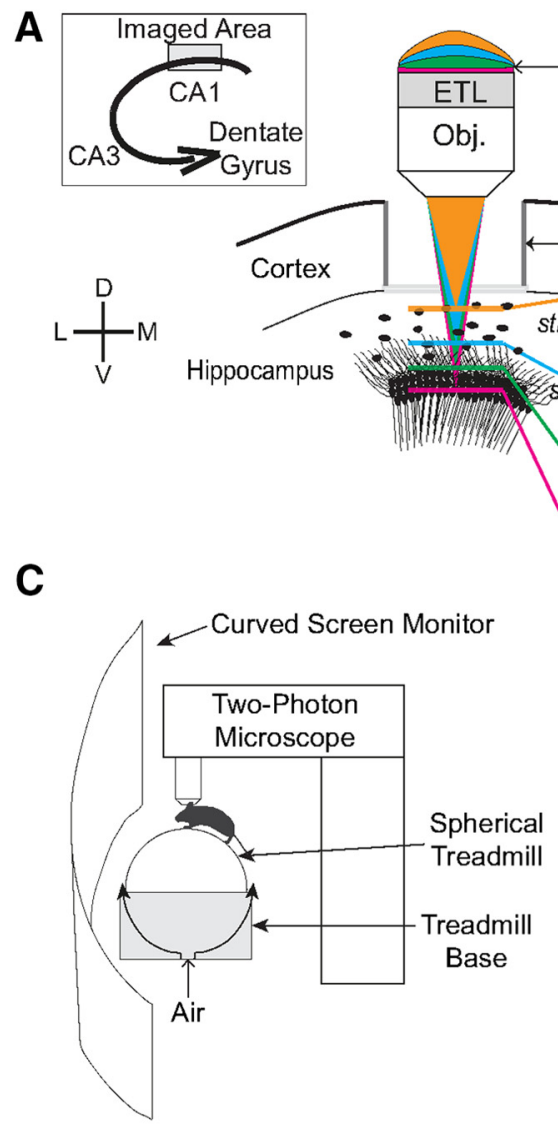

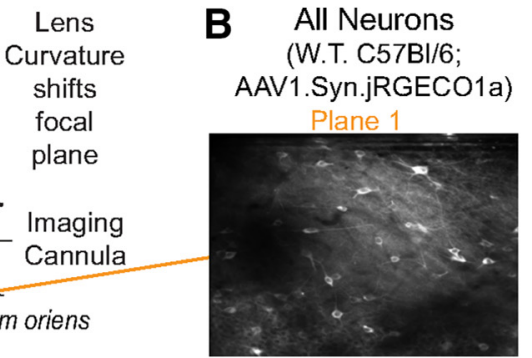

Plane 2

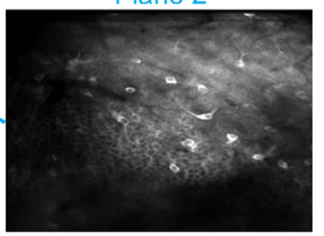

Plane 3

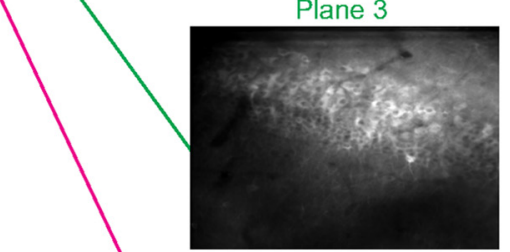

Plane 4

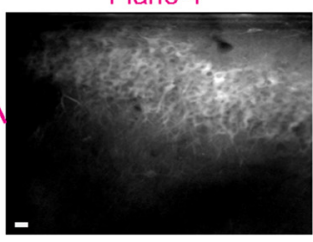

Depth Somatostatin Interneurons

(relative (SST-cre +l-;

to AAV1.Flex.Syn.GCaMP6f

Plane1)

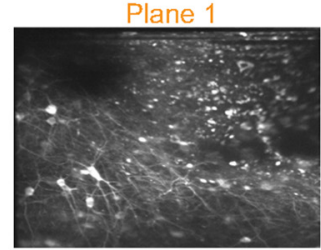

Plane 2

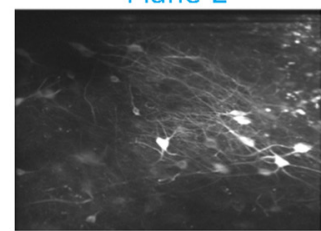

Plane 3

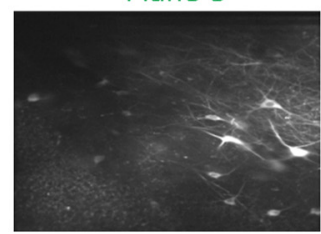

Plane 4

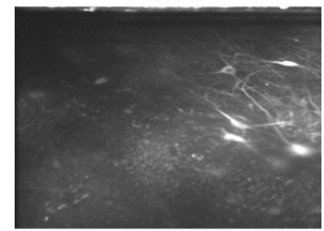

Time

$65 \mathrm{~ms}$

$129 \mathrm{~ms}$

$194 \mathrm{~ms}$

$258 \mathrm{~ms}$

Figure 1. Experimental setup. $\boldsymbol{A}$, Schematic of imaging configuration. Inset, Schematic of imaged area in CA1. ETL rapidly switches focal plane. Different curvatures of the ETL alter laser divergence resulting in different focal plane depths (represented by different colors). For simplicity, the ETL is shown above the objective. In reality, it is in the excitation light path, before the laser scanners, and does not impinge on the collection pathway. B. Sample in vivo imaging planes from animals with all neurons labeled with AAV-Syn-jRGECO1a or somatostatin interneurons labeled with cre-dependent AAV-Flex-Syn-GCaMP6f in somatostatin-cre mice. Four planes are imaged sequentially starting with the most dorsal plane and this sequence repeats until the end of the imaging session. Time shown is the elapsed time for one imaging cycle. Scale bar, $20 \mu \mathrm{m}$. C, Schematic of VR set up. Head-fixed mice can run freely on a spherical treadmill floating on a cushion of air. Ball movement is tracked by an optical computer mouse (data not shown) and fed into the VR engine, which updates the visual scene on a curved-screen computer monitor positioned in front of the animal.

using the "regionprops" Matlab function. Eccentricity ranges from 0 to 1 , with a perfect circle having eccentricity of 0 and more elliptical shapes toward 1.

Experimental design and statistical analysis. For pyramidal neurons, we recorded 479 cells (average \pm SD: $119.8 \pm 39.1$ cells per animal; range: 89-177 cells) from four male mice. We recorded 192 somatostatin-crepositive cells (average \pm SD: $28.4 \pm 22.3$ per animal; range: $18-73$ cells) from five mice (three males, two females). We recorded 125 parvalbumin-cre-positive cells (average \pm SD: of $28.0 \pm 11.3$ per animal; range: $8-34$ cells) from five mice (four males, one female).

Cell-to-cell $\Delta F / F$ and cell $\Delta F / F$-to-speed correlations were calculated using Pearson's correlation coefficient and significance was corrected for multiple comparisons using a Bonferroni-Holm correction. LMI significance was calculated by bootstrapping $1 \mathrm{~s}$ bins, sampled at $\leq 2 \mathrm{~s}$ intervals (Pakan et al., 2016). Significance of differences was calculated using Wilcoxon rank-sum tests for unpaired data and Wilcoxon signedrank tests for paired data. Multiple comparisons were Bonferroni corrected as indicated. Statistical analyses were performed in Matlab (RRID:SCR_001622).

Phase angle of cross-correlation between $\Delta F / F$ and ball speed was calculated using the Hilbert transform. The Hartigan dip test is commonly used to test for bimodality but has little power when the two populations have uneven sizes, as is the case here. To test for a diametrically bimodal population (phase angle difference of $180^{\circ}$ between populations), we doubled the phase angle of each cell and used the Rayleigh test for uniformity on the resulting distribution (Zar, 2007). For exam- ple, if there are two population peaks at phase angles of $90^{\circ}$ and $270^{\circ}$, doubling the phase angles results in one peak at $180^{\circ}\left(90^{\circ} \times 2=180^{\circ}\right.$; $\left.270^{\circ} \times 2=540^{\circ}=180^{\circ}\right)$. If the phase-doubled distribution is unimodal (by Rayleigh test), the initial distribution is significantly bimodal. The Rayleigh test was implemented from CircStat for Matlab (Berens, 2009). There was strong correspondence between cells classified by correlation of activity to speed and phase angle. For somatostatin interneurons, 127 of 128 cells $(99.2 \%)$ of positively correlated cells were classified in the positive phase while 31 of $32(96.9 \%)$ negatively correlated cells were classified in the negative phase. There was similar correspondence for parvalbumin cells. We classified 48 of 51 cells $(94.1 \%)$ of positively correlated cells in the positive phase and 10 of 11 (90.9\%) negatively correlated cells in the negative phase. Throughout this paper, we use activity-to-speed correlation to characterize cells since only a subset of cells are classified by phase angle (experiments with $>10$ start-stop transitions: 166 of 192 somatostatin neurons and 64 of 125 parvalbumin neurons). Kernel density estimate was calculated by convolving each data point with a kernel function and summing the resulting curves to yield a probability density function. We used a Gaussian kernel with an SD (bandwidth) of $11.6^{\circ}$, which was the estimated optimal bandwidth for this dataset, as calculated by the Matlab function ksdensity. Stability of correlations over days was assessed via Pearson correlation coefficients by measuring the extent to which cell correlation with speed in one recording session correlated with subsequent sessions. All data are presented as \pm SEM unless otherwise noted. 
A

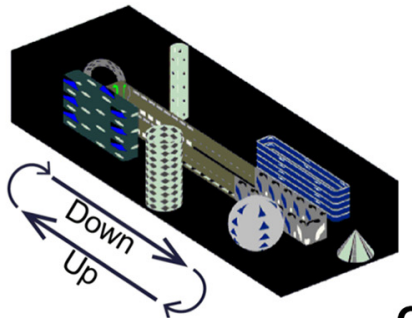

\section{B}

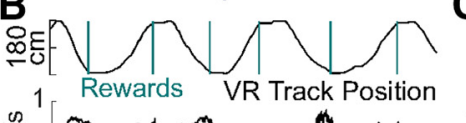

क ${ }^{1}$ Rewards VR Track Position
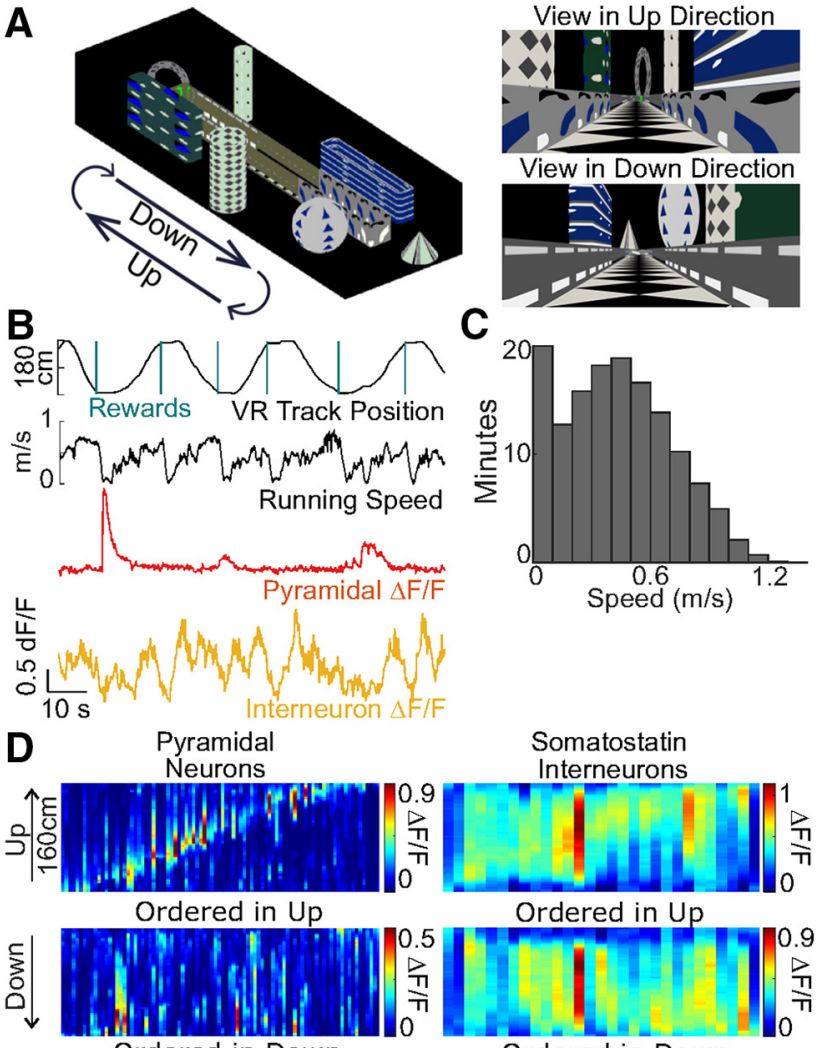

View in Down Direction

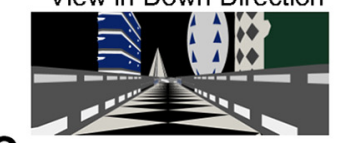

C
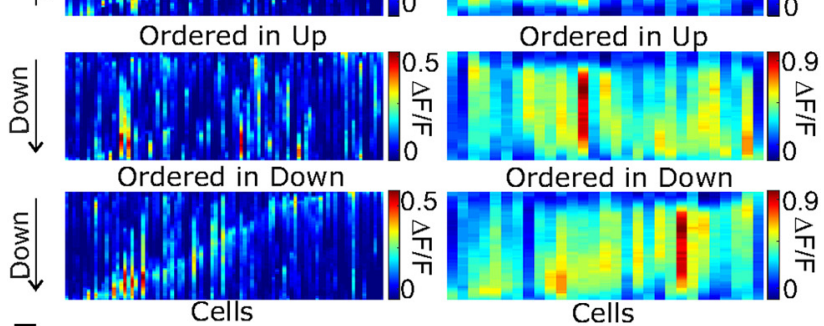

Ordered in Down

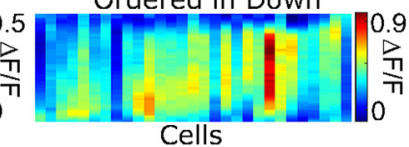

E

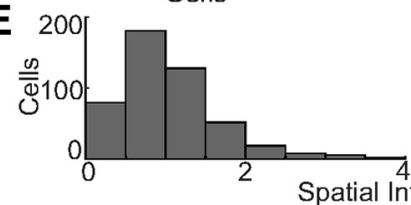

$\mathbf{F}$

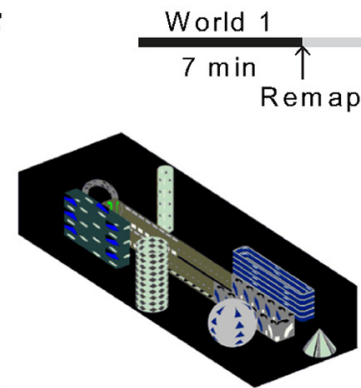

G

World 1

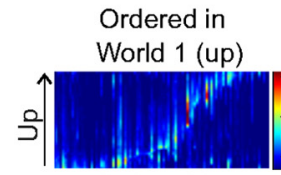

Ordered in

World 1 (down)
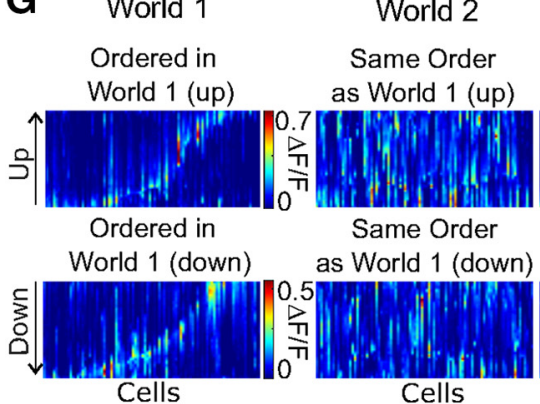

World 2

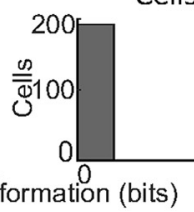

World 2

$14 \mathrm{~min}$
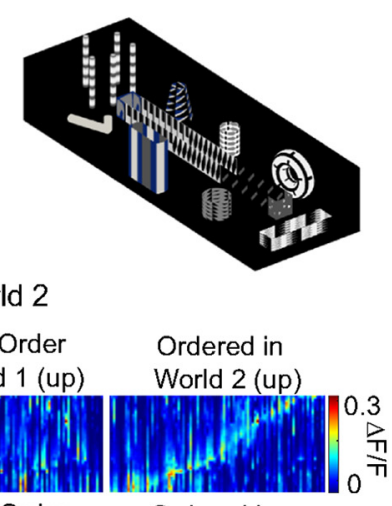

Ordered in

World 2 (down)

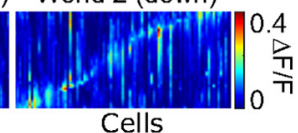

Figure 2. Spatial activity of neurons and remapping in VR. A, Overview of VR track. Mice run to one end of the VR track and then to the opposite end to receive water rewards. Mice are restricted to the central corridor surrounded by local wall cues and distal landmarks. Arrows indicate up-direction and down-direction runs. Right, Monitor views in up and down directions.

\section{Results}

\section{Characterization of neuronal calcium activity in VR}

track running

To investigate in vivo neural network dynamics, we used twophoton imaging of neuronal calcium activity during a spatial navigation task in VR (Dombeck et al., 2010). To monitor a larger fraction of the network, as well as record from cells along the deep to superficial axis of the hippocampus, the microscope was fitted with an ETL to rapidly modulate the divergence of laser light through the objective, allowing fast $z$-axis focal jumps (Grewe et al., 2011). This enabled us to capture sequential imaging frames moving from dorsal to ventral at a total range of $\leq 180 \mu \mathrm{m}$ (Fig. $1 A)$. In most experiments, we imaged four separate dorsalventral planes at a rate of $4 \mathrm{~Hz}$ in each plane. Before behavioral training, mice were injected with AAV to express a genetically encoded calcium sensor targeted unilaterally to the CA1 region of the dorsal hippocampus. AAV1-Syn-jRGECO (Dana et al., 2016) was injected in WT mice to label all neurons. A mixture of AAV1CaMKII-cre and AAV1-Syn-Flex-GCaMP6f (Chen et al., 2013) was injected in WT mice to label pyramidal neurons. AAV1-SynFlex-GCaMP6f was injected into somatostatin-cre ${ }^{+/-}$transgenic mice (Taniguchi et al., 2011) to label somatostatin-expressing interneurons (Fig. 1B). An imaging cannula was implanted to allow imaging into the hippocampus (Fig. 1A). Head-fixed mice ran on a floating spherical treadmill (Styrofoam ball) to control movement through the VR visual environment displayed on a curved-screen monitor in front of the animal (Fig. 1C). Ball movement was tracked with an optical computer mouse and fed into the VR engine to update the visual scene.

To compare the properties of neuronal activity in our VR setup to previous real-world and VR experiments, we used calcium transients as a proxy for spiking and examined spatial coding in pyramidal and somatostatin interneurons. We measured calcium activity from pyramidal neurons in CaMKII-cre/SynFlex-GCaMP6f-injected ( $N=2$ mice, $n=281$ cells $)$ and Syn-

$B$, Data collected during VR behavior. Mouse position in VR track and timing of water rewards (transients). Ball speed during VR task. $\Delta F / F$ of jRGECO1a-labeled pyramidal neuron. $\Delta F / F$ of simultaneously recorded jRGEC01a-labeled interneuron. C, Binned distribution of ball speed for five mice during VR behavior. $D$, Top, Left, Heatmap of pyramidal neuron mean $\Delta F / F$ versus track position, specifically for up-direction runs. Each column represents the activity of an individual neuron. To generate the heatmap, $\Delta F / F$ in each spatial bin was summed and divided by occupancy time. Cell order is sorted by track position of peak $\Delta F / F$. Reward zones at the ends of the track have been excluded. Top, Right, From a separate experiment, heatmap of somatostatin interneuron mean $\Delta F / F$ versus track position. Middle, Left, Heatmap of pyramidal neuron activity in down-direction runs, but with cell order set by up-direction runs. Note that spatial pattern of activity is lost, indicating direction-sensitive spatial activity of pyramidal neurons in this VR task. Middle, Right, Heatmap of somatostatin interneuron activity in down-direction runs, but with cell order set by up-direction runs. Bottom, Left, Pyramidal neuron heatmap in down-direction runs and sorted by position of peak $\Delta F / F$. Bottom, Right, Somatostatin interneuron heatmap in down-direction runs and sorted by position of peak $\Delta F / F$. E. Left, Distribution of spatial information from 479 pyramidal neurons in four mice. Right, Distribution of spatial information from 192 somatostatin interneurons from five mice. $\boldsymbol{F}$, Timeline of remapping experiment. Animals spend 7 min in World 1 , which is then instantaneously replaced with World 2, where they run for an additional $14 \mathrm{~min}$. Data shown is after $4-5 \mathrm{~d}$ training in the remapping paradigm so animals are familiar with World 2. G, Top, Left, World 1 heatmap of pyramidal neuron mean $\Delta F / F$ versus track position, in up-direction runs. Top, Middle, World 2 heatmap for up-direction runs with same cell order as World 1. Note that spatial activity of neurons in World 1 is not maintained in World 2, indicating remapping. Top, Right, World 2 heatmap with cells sorted by World 2 spatial activity. Bottom, Left, World 1 heatmap of pyramidal neuron mean $\Delta F / F$ versus track position in down-direction runs. Bottom, Middle, World 2 heatmap in down-direction runs with same cell order as World 1. Bottom, Right, World 2 heatmap with cells sorted by World 2 spatial activity. 
jRGECO-injected ( $N=2, n=198$ cells) mice. To identify putative pyramidal neurons from pan-neuronal jRGECO1a labeling, we eliminated putative interneurons based on anatomy and calcium dynamics (see Materials and Methods). Movies were corrected for brain motion and fluorescence time-series for individual cells were extracted from ROIs selected using an automated cell-segmentation algorithm (Mukamel et al., 2009). Significant calcium transients in pyramidal neurons were detected with an algorithm designed to give $<5 \%$ false-positive error rates (Dombeck et al., 2009) and used for subsequent analysis. We did not extract significant calcium transients from interneuron activity traces. In contrast to hippocampal pyramidal neurons, which have a very low rate of spontaneous firing, most interneurons fire spontaneously and this basal activity is modulated up and down during behavior (Ranck, 1973; Ego-Stengel and Wilson, 2007; Royer et al., 2012; Varga et al., 2012; Katona et al., 2014). Thus, the continuous $\Delta F / F$ trace of calcium activity is more representative of interneuron activity than a thresholded version. Interneuron ROIs were identified with a semiautomated procedure and visually inspected for fluctuations in $\Delta F / F$.

Mice were water-scheduled and trained to run back and forth between ends of a $180 \mathrm{~cm}$ virtual track (Fig. 2A) for water rewards, while we recorded calcium activity from neurons (Fig. $2 B$ ). Mice ran in a short burst to traverse the track, stopped to drink the water reward, turned the ball to turn around in VR, and ran to the other end for another water reward. The distribution of ball speed shows a minimum at $15 \mathrm{~cm} / \mathrm{s}$, which we used as a threshold for defining stopped and moving periods. Average ball speed was $28.2 \pm 2.4 \mathrm{~cm} / \mathrm{s}$ overall, $35.3 \pm 2.3 \mathrm{~cm} / \mathrm{s}$ during movement periods, and $7.5 \pm 1.1 \mathrm{~cm} / \mathrm{s}$ during stopped periods $(N=5$ mice).

For each neuron, we generated a spatial activity map by binning the track, summing the neuron's $\Delta F / F$ in each bin, and normalizing by occupancy. Cell order for display was sorted by track position of peak $\Delta F / F$, progressing from the "bottom" to the "top" of the VR track (Fig. 2D, top and bottom). Because place fields are direction specific in track running tasks, the updirection and down-direction runs were analyzed separately (McNaughton et al., 1983). This directionality can be seen when the spatial activity of cells, as sorted by track position activity in up-direction runs, is shown for down-direction runs (Fig. 2D, left, top and middle). If spatial activity of pyramidal neurons was not directional, these two maps would look similar. In contrast, the spatial activity in down-direction runs is dissimilar, indicating direction-specific spatial activity in pyramidal neurons during VR track running.

We found that pyramidal neurons had more spatially restricted activity fields than interneurons (Fig. $2 B, D, E$, left and right; pyramidal activity field width, $74.77 \pm 0.47 \mathrm{~cm}, N=4$ mice, $n=479$ cells; somatostatin interneuron firing activity width, $91.25 \pm 0.92 \mathrm{~cm}, N=5, n=192, p=5 \times 10^{-137}$; Wilcoxon rank sum). To quantify space-coding properties across the pyramidal population, we calculated spatial information for individual cells and plotted the resulting distributions. Pyramidal neurons showed a skewed distribution of spatial information with a median of 0.75 and mean of $0.98 \pm 0.06$ bits (Fig. 2E, left), which was qualitatively similar to other distributions in realworld and VR tasks (Jung et al., 1994; Aghajan et al., 2015). Interneurons had a median of 0.11 and mean of $0.13 \pm 0.01$ bits (Fig. $2 E$, right), encoding much less spatial information than pyramidal neurons ( $p=4 \times 10^{-147}$; Wilcoxon rank sum). These data show that during VR behavior, pyramidal neurons encode more spatial information and have smaller activity fields than interneurons, similar to pyramidal neuron activity in freely moving animals (Wilson and McNaughton, 1993).

Place fields can undergo global remapping in different environments: cells can lose existing place fields, gain new place fields, or move their place-specific firing. To determine whether hippocampal place representations are environment-dependent in our VR task, we trained animals in another visual environment (World 2) and compared the spatial activity for the same cells in World 1 and World 2 (Fig. 2F). Upon switching to World 2, the map of spatial-specific activity from World 1 was lost, indicating remapping of place fields (Fig. 2G). Spatial information in pyramidal neuron activity in both environments was comparable and much larger than spatial information in interneurons (spatial information was slightly greater in World 1 ; median of 0.52 and mean of $1.36 \pm 0.09$; World 2 , median of 0.46 and mean of $0.65 \pm$ $0.03 ; N=3, n=372, p=0.005$, Wilcoxon rank sum). Together, these data show place-specific activity and environment-dependent remapping of hippocampal pyramidal neurons in our headfixed VR task, similar to previous results in VR (Aronov and Tank, 2014; Cohen et al., 2017) and freely moving animals (Muller and Kubie, 1987; Leutgeb et al., 2004).

\section{Correlated and anticorrelated activity in somatostatin interneurons}

To look for possible state-dependent modulation of activity as animals performed the VR track running task, we searched for widespread temporal correlations in activity across cells within the pyramidal and interneuron populations. We recorded calcium activity from the molecularly defined somatostatin-expressing class of interneurons and found that many cells showed synchronized periodic activity fluctuations. Notably, however, a subset of cells had activity that was anticorrelated to the majority of the interneuron population (Fig. $3 A, B$ ). For each experiment, we generated a matrix of pairwise neuron-neuron activity correlation values and then plotted the distribution of all correlation $r$ values (Fig. 3C,D). After pooling the correlation $r$ values from all pairs of somatostatin interneurons into a single distribution, 1810 pairs (72.8\%) were positively correlated, 329 pairs (13.3\%) were negatively correlated, and 341 pairs (13.8\%) had no significant correlation (Fig. $3 E ; N=5$ mice, $n=192$ cells; $p<0.05$, Pearson correlation coefficient with Bonferroni-Holm correction for multiple comparisons), indicating that a majority of somatostatin interneurons have correlated activity during VR track running, with a small percentage of anticorrelated neurons.

When we examined the correlation structure of pyramidal neuron population activity during VR track running, there was much lower activity correlation across cells, as can be seen in raw $\Delta F / F$ traces (Fig. $3 F, G$ ), in the correlation matrix of neuronneuron activity correlation values, and in the distribution of correlation $r$ values (Fig. $3 \mathrm{H}, I$ ). In the pooled distribution of correlation $r$ values for all pyramidal neurons, $3716(21.0 \%)$ pairs were positively correlated, $5648(14.6 \%)$ pairs were negatively correlated, and 24,888 (71.0\%) pairs were not significantly correlated (Fig. 3J; $N=$ four mice, $n=479$ cells; $p<0.05$, Pearson correlation coefficient with Bonferroni-Holm correction for multiple comparisons). Thus in strong contrast to the pyramidal neuron population, which shows relatively little correlated population activity, most somatostatin neurons have similar temporal patterns of activity, with a small percentage of cells whose activity is anticorrelated to the rest of the population. 
Locomotion-activated and immobilityactivated somatostatin interneurons

To investigate the locomotor correlates of neuronal activity, we aligned $\Delta F / F$ traces with the movement activity of the mouse, as measured by ball speed (Fig. $4 A, B$ ). Many cells were more active during movement, although some showed the opposite pattern, becoming more active at low speeds. To parametrically describe the relationship between activity and movement, we measured the correlation between $\Delta F / F$ and ball speed for each cell (Fig. 4A, B, sample cell correlograms from two mice; Fig. $4 C$, $r$ values of correlation for all cells from two mice). The distribution of $r$ values from all mice (Fig. $4 D ; N=$ 5 mice) show consistency across animals, with activity in most cells correlated with speed, while a small percentage was anticorrelated. To quantify, we pooled the $r$ values from all somatostatin interneurons into a single distribution and found activity in $74.5 \%$ of neurons was correlated with speed, activity in 18.5\% of neurons was anticorrelated with speed, and $6.8 \%$ of neurons had no correlation (Fig. $4 E ; N=5$ mice, $n=192$ cells; $p<0.05$, Pearson correlation coefficient with Bonferroni-Holm correction for multiple comparisons). We further quantified the influence of locomotion on cellular activity by calculating an LMI for individual cells. In general, LMI measures were similar to the activity-to-speed correlation. Directly comparing the significance of speed/fluorescence correlation to LMI measures for all cells, we found $84.7 \%$ (150 of 177) of cells were significant in both measures and $15.3 \%$ (25 of 177) of cells significant by LMI only. Together with Figure 1, these data show that many somatostatin interneurons have correlated activity occurring during locomotion. A smaller fraction is active during immobility and this activity is anticorrelated to activity in most of the population.

To further characterize the organization of activity by movement, we identified transitions between immobility and movement (based on the $15 \mathrm{~cm} / \mathrm{s}$ speed threshold calculated from the distribution of animal movement in Fig. 2C) to align starttriggered and stop-triggered rasters of neuronal activity. A representative experiment from a behavioral session with 41 immobile periods is shown in Figure $5 A-C$. Speed on every trial is shown in Figure $5 A$. In start-triggered events, the activity of a cell with positive correlation between activity and movement showed a sharp increase at the beginning of movement (Fig. 5B, left), whereas neuronal activity of a negatively correlated cell shows a sharp drop (Fig. 5C, left). Conversely in stop-triggered events, this positively correlated cell showed a sharp drop in activity when transitioning to immobility (Fig. $5 B$, right), whereas this negatively correlated cell increased activity (Fig. $5 C$, right). We used the same speed as a dividing line for locomotion and immobility $(15 \mathrm{~cm} / \mathrm{s})$ in both start-triggered and stop-triggered events. While this threshold matches the transition of activity in start-triggered events (Fig. 5B, C,E, left), the locomotion threshold for stop-triggered events may be better fit by shifting the threshold to the left, corresponding to a higher speed (Fig. $5 B, C, E$, right).

The average fluorescence time series across all trials of the two example cells, plotted along with the average speed, shows that changes in speed are closely matched by changes in fluorescence in the positively correlated cell, while activity in the negatively correlated cell decreases with speed (Fig. 5D). Next, we looked at start-triggered and stop-triggered activity in the somatostatin population. We only included experiments where the animal had $>10$ start/stop episodes that crossed our speed threshold of $15 \mathrm{~cm} / \mathrm{s}$ with stop duration of $>1$ s (166 of 192 somatostatin neurons). The average activity of these somatostatin interneurons at locomotion transitions shows that activity in most cells increases during movement while activity in a small percentage of cells is inhibited (Fig. 5E). We quantified this relationship by 


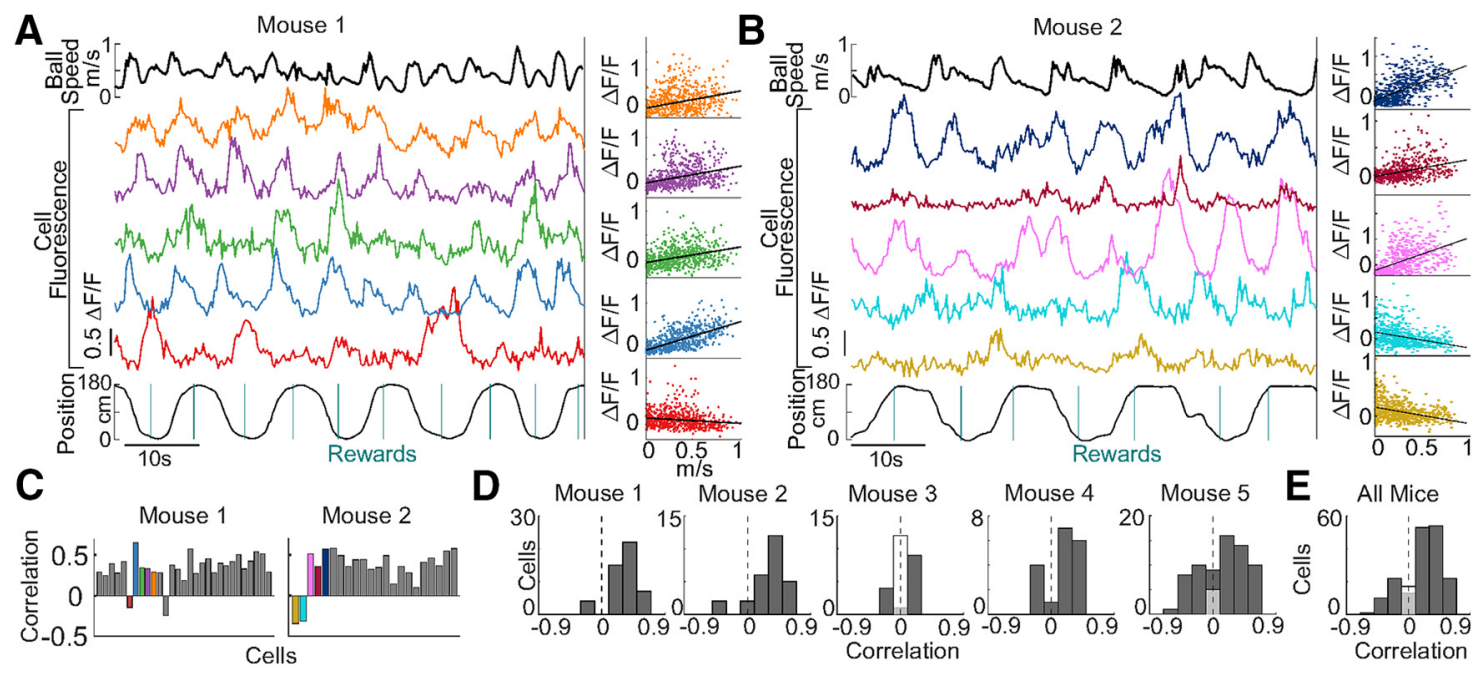

Figure 4. Locomotion-activated and immobility-activated somatostatin interneurons. $A, \Delta F / F$ of five somatostatin interneurons from a single imaging plane, plotted with ball speed (top), VR position, and rewards (bottom). Right, Correlation plot of $\Delta F / F$ versus ball speed (binned to match the frame rate of imaging) for cells shown in $A$ for entire imaging session. Line is linear regression. $\boldsymbol{B}$, Five somatostatin interneurons from a single imaging plane from another mouse. $\boldsymbol{C}, \Delta F / F$-to-speed correlations for all cells from two mice shown in $\boldsymbol{A}$ and $\boldsymbol{B}$. Colored bars match individual cells shown in $\boldsymbol{A}$ and $\boldsymbol{B}$. Solid bar (either dark gray or colored) is a significant relationship, either correlation or anticorrelation. $\boldsymbol{D}$, Distribution of activity-to-speed correlation $r$ values for neurons in individual somatostatin-cre mice. $E$, Pooled distribution of activity-to-speed correlation $r$ values for all somatostatin interneurons.

comparing the fluorescence between immobile and moving periods (baseline period was -3 to $-1 \mathrm{~s}$ before transition and postperiod was $1-3 \mathrm{~s}$ after mobility transition). Here we split cells into positively and negatively correlated cells based on their correlation between activity and locomotion. We found that positively correlated cells increased activity going from immobile to moving (Fig. $5 F$, left; $n=133$ cells; $0.10 \Delta F / F \pm 0.01$ to $0.20 \pm 0.01$, $\left.p=1.9 \times 10^{-11}\right)$ and decreased activity when going from moving to immobile $\left(0.12 \Delta F / F \pm 0.01\right.$ to $-0.07 \pm 0.01, p=1.2 \times 10^{-22}$, Wilcoxon signed rank). Conversely negatively correlated cells decreased activity from moving to immobile (Fig. $5 F$, right; $n=33$ cells; $0.18 \pm 0.03$ to $\left.0.05 \pm 0.01, p=1.7 \times 10^{-5}\right)$ and increased activity when going from moving to immobile $(-0.01 \Delta F / F \pm$ 0.01 to $\left.0.13 \pm 0.02, p=4.2 \times 10^{-7}\right)$. Thus, when ongoing behavior is parsed into higher resolution stop-triggered and start-triggered events, two populations of functionally defined somatostatin interneurons can be identified.

Next, we examined the temporal relationship between movement and activity by generating a cross-correlation of speed and activity signals (cross-correlation for sample cell used in Fig. 5A-D shown in Fig. 6A). We plotted the cross-correlation function as a heat map for all somatostatin cells and found that in positively correlated cells, activity followed speed with a short delay (Fig. 6B; $0.57 \pm 0.08 \mathrm{~s}, 133$ neurons). Activity in negatively correlated cells was much more delayed relative to speed (Fig. $6 B$; $4.1 \pm 0.16 \mathrm{~s}, 33$ neurons) and instead the nadir of activity slightly preceded speed $(-0.32 \pm 0.2 \mathrm{~s})$. The $\sim 4 \mathrm{~s}$ delay between speed and $\Delta F / F$ in negatively correlated cells reflects the average latency between movement and a subsequent immobile period (when negatively correlated cells would again become active).

These data demonstrate that the activity of individual somatostatin interneurons can exhibit a positive or negative correlation with movement. To test whether the population is bimodal, we analyzed the temporal relationship between activity and speed in start-triggered trials (Fig. 5E, left) using circular statistics by extracting the phase angle of the cross-correlation at zero time lag and plotting the distribution of cells (Fig. 6C). The resulting distribution shows a statistically significant diametrically bimodal population; one population displays activity that peaks with lo- comotor speed, whereas the second population is inhibited with speed (phase angle difference of $180^{\circ}$ as tested with Rayleigh $z$ test between the original distribution and the distribution with all angles doubled, $\left.p=6.6 \times 10^{-20}\right)$. To further visualize bimodality, we made a linear distribution of phase angle by "folding over" the rose plot along the line $0-180^{\circ}$ (phase angle doubles back from 180 to $360^{\circ}$; Fig. $6 D$ ). The resulting histogram was smoothed by a kernel density estimate to visualize the underlying distribution by minimizing the effect of histogram bin sizes (Fig. $6 D$, right axis). These data show that somatostatin interneurons can be split into two populations, one whose activity peaks close to the peak of speed, and another whose activity is shifted by $180^{\circ}$ so that the nadir of activity occurs during peak speed.

We also investigated the possibility that interneuronal calcium activity was more strongly tied to other movement-related variables than total ball speed. For each cell, we calculated goodness of fit using residual sum of squares to the linear regression of fluorescence to total ball speed, ball forward speed, ball yaw speed (total ball speed is the combination of forward and yaw, or rotational, speed), VR speed (in the long axis of the track), ball acceleration, and VR acceleration. All speed variables exhibited a better fit of linear regression to activity than acceleration variables $(N=5$ mice, $n=192$ cells; mean $r^{2}$ s: total ball speed, $0.103 \pm 0.008$; forward ball speed, $0.092 \pm 0.007$; yaw ball speed, $0.091 \pm 0.009$; VR speed, $0.098 \pm 0.007$; ball acceleration, $0.007 \pm 0.002$; VR acceleration, $0.006 \pm 0.001)$. Thus, total ball speed showed the best correlation with activity in somatostatin interneurons.

Locomotion is correlated to activity in most cells in the somatostatin interneuron population and anticorrelated to activity in a small subset; however, immobile periods mostly occur after rewards, leaving open the possibility that activity is governed by reward rather than movement. To distinguish between the influence of rewards and locomotion, we examined immobile periods that occurred $>4 \mathrm{~s}$ after reward delivery, as well as immobile periods that occurred in the track rather than in the reward zones (Fig. 7A). During nonreward periods, there was still a significant increase in activity with locomotion in positively correlated cells (Fig. $7 B ; 82$ neurons, $-0.05 \Delta F / F \pm 0.01$ to $0.05 \pm 0.01, p=1.2 \times$ $10^{-20}$ ) and a decrease in negatively correlated cells (16 neurons, 
A
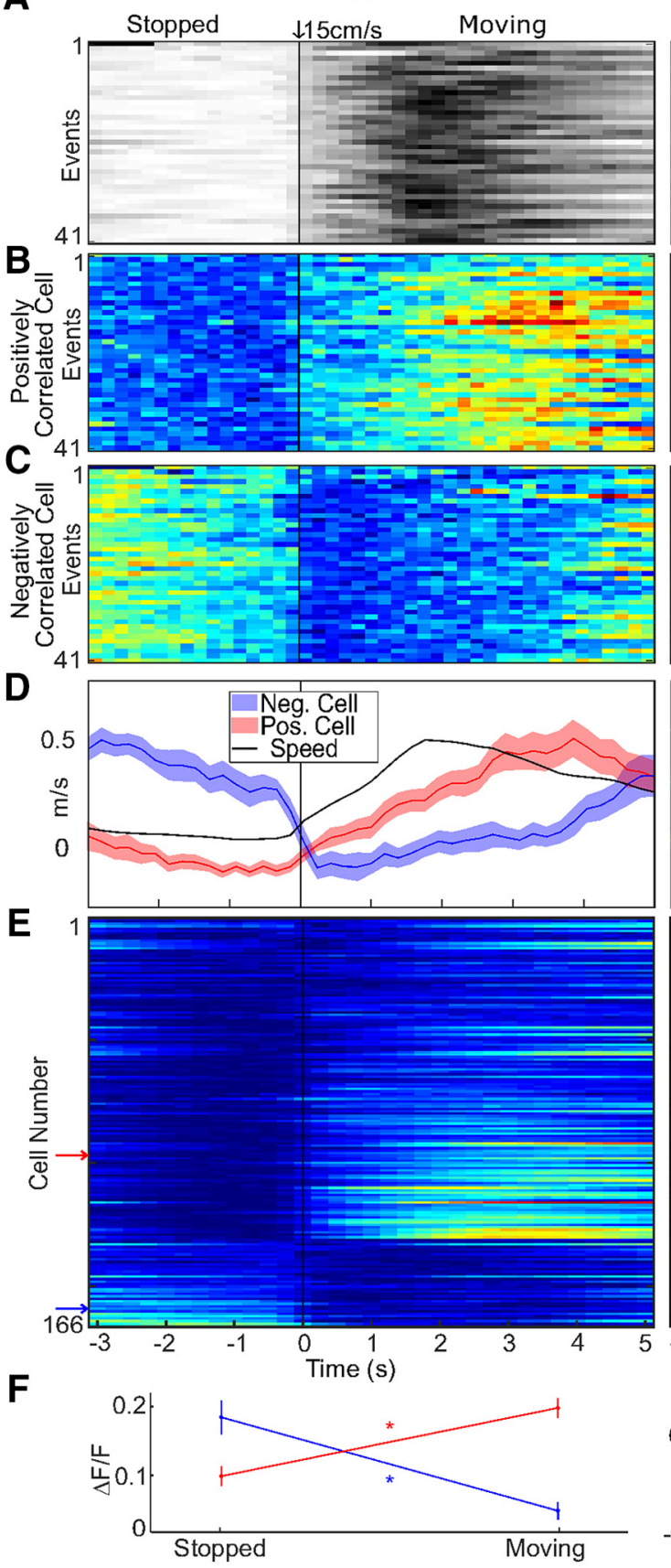

Stop-Triggered
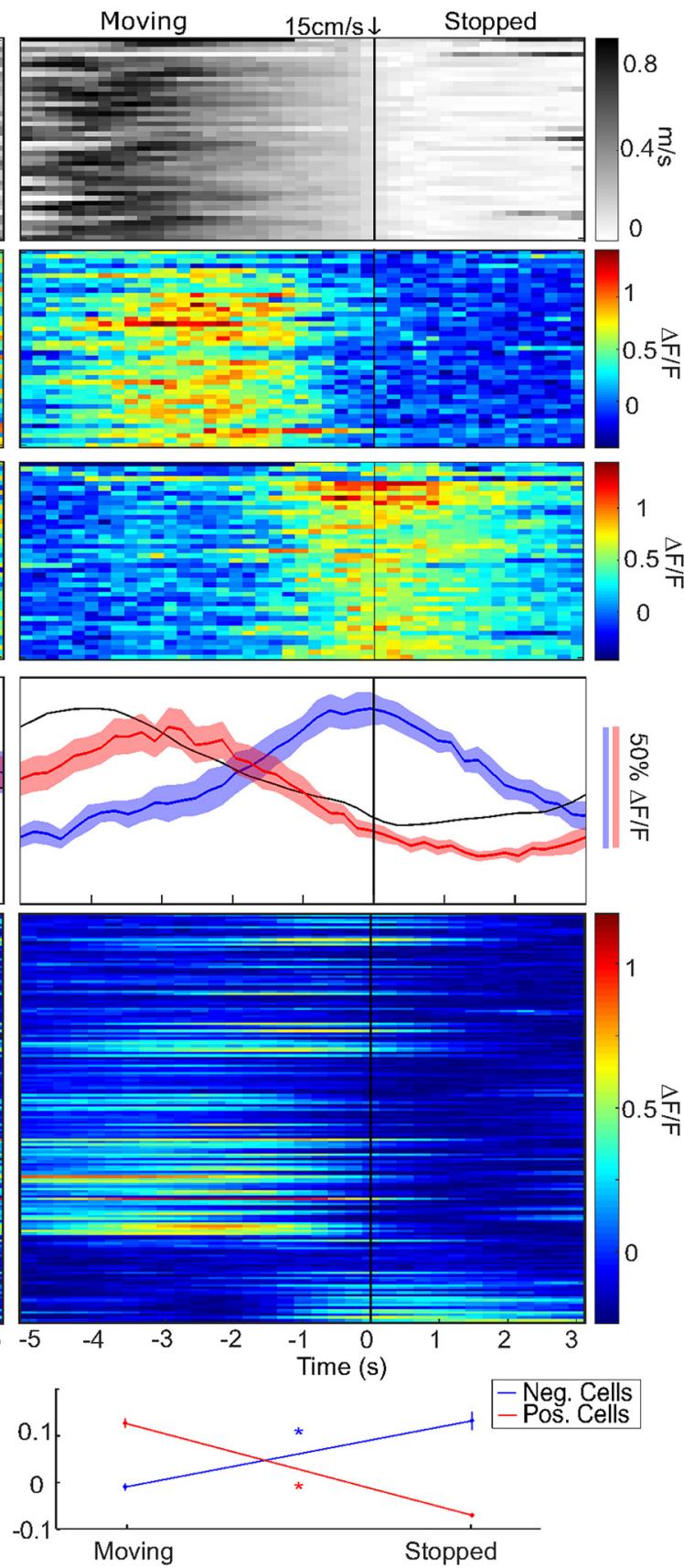

Figure 5. Somatostatin interneuron activity is organized at locomotion transitions. $\boldsymbol{A}$, Transitions between immobility and movement were identified and used to align locomotion starttriggered and stop-triggered events in one experiment. Shown is the heatmap of ball speed over time for each detected event in one experiment. Left, Start-triggered events (immobile to moving). Right, Stop-triggered events (moving to immobile). Black line running through $\boldsymbol{A}-\boldsymbol{E}$ marks time of locomotion transition. The same speed threshold was used for start-triggered and stop-triggered events ( $15 \mathrm{~cm} / \mathrm{s}$ ). $\boldsymbol{B}, \Delta$ F/Fheatmap for each start-triggered (left) or stop-triggered (right) event from a single cell with a positive activity-to-speed correlation. $\boldsymbol{C}, \Delta F / F$ heatmap for each start-triggered (left) or stop-triggered (right) event from a cell with a negative activity-to-speed correlation. $\boldsymbol{D}$, Line plot of average $\Delta$ F/F over all events for cells in $\boldsymbol{B}$ and $\boldsymbol{C}$, plotted together with ball speed. Shaded area is SEM. $\boldsymbol{E}$, Heatmap of mean $\Delta F / F$ over time during start-triggered and stop-triggered events for somatostatin interneurons from experiments with $>10$ start-stop transitions (166 of 192 neurons). Cells with positive activity-to-speed correlation are shown at top and negative activity-to-speed correlation at bottom. Within each class, neuron order is sorted from low to high magnitude of correlation or anticorrelation. Neuron order is the same for start-triggered and stop-triggered heatmaps. Red and blue arrows mark positively correlated cell shown in $\boldsymbol{B}$, and negatively correlated cell in $\boldsymbol{C}$, respectively. $\boldsymbol{F}$, Comparison of mean $\Delta F / F$ ( \pm SEM) between moving and stopped periods for all positively (red) and negatively (blue) correlated cells. ${ }^{*} p \leq 1.7 \times 10^{-5}$.

$0.11 \pm 0.02$ to $\left.0.03 \pm 0.01, p=6.7 \times 10^{-5}\right)$, indicating that activity is tied to locomotion rather than reward.

Positively and negatively correlated parvalbumin interneurons

These data demonstrate that $18.5 \%$ of somatostatin interneurons have activity that increases during immobility. Is this functionally identified subset exclusive to somatostatin interneurons, or do other genetically specified interneuron populations also exhibit this activity? To investigate this question, we recorded from another population of interneurons, the soma-targeting parvalbuminexpressing interneurons. Similar to activity in the somatostatin interneurons, activity in most parvalbumin interneurons was positively correlated with movement, although there was a small 
A Start-Triggered
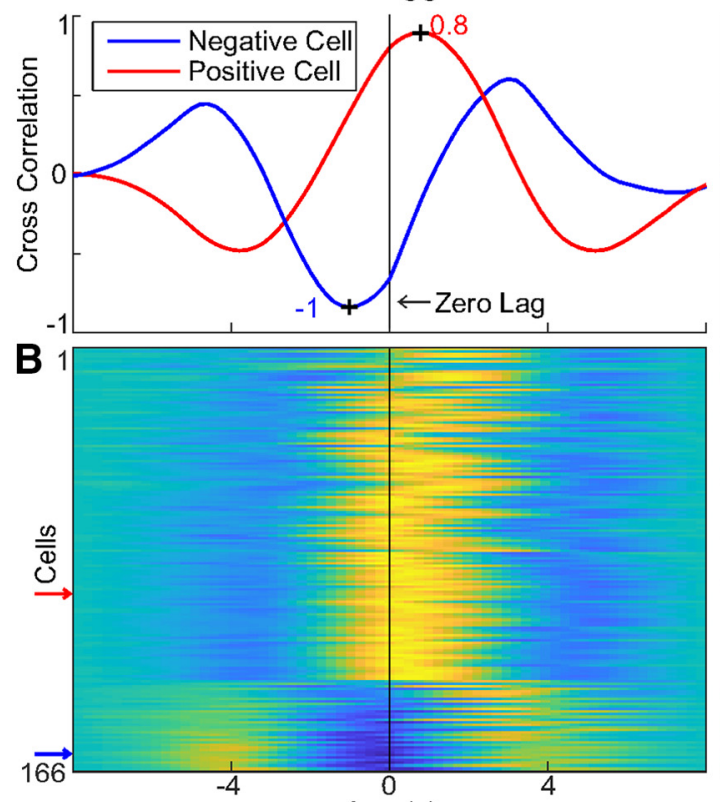

$\operatorname{Lag}(\mathrm{s})$

C

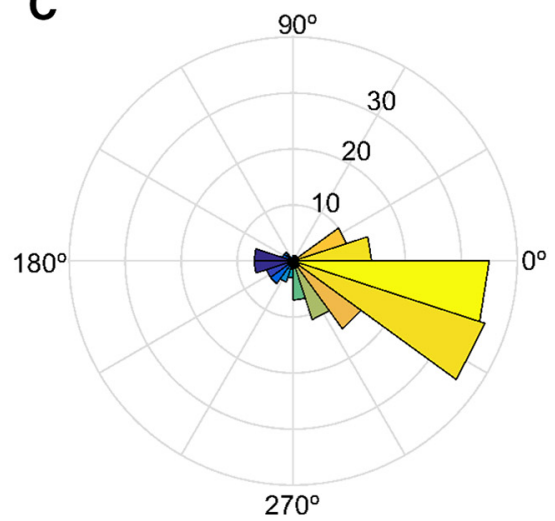

Stop-Triggered
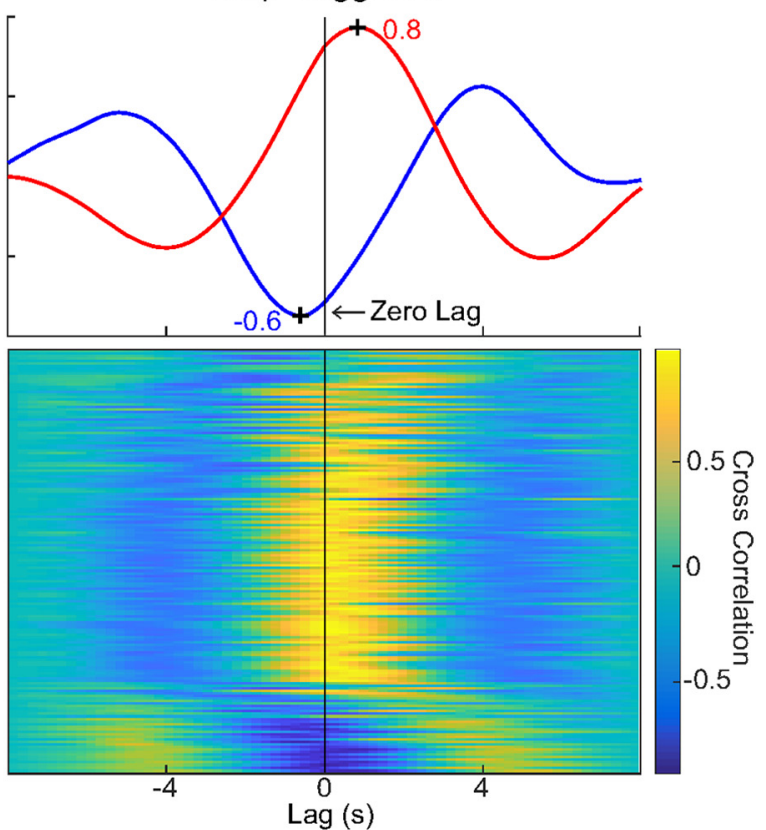

D 40

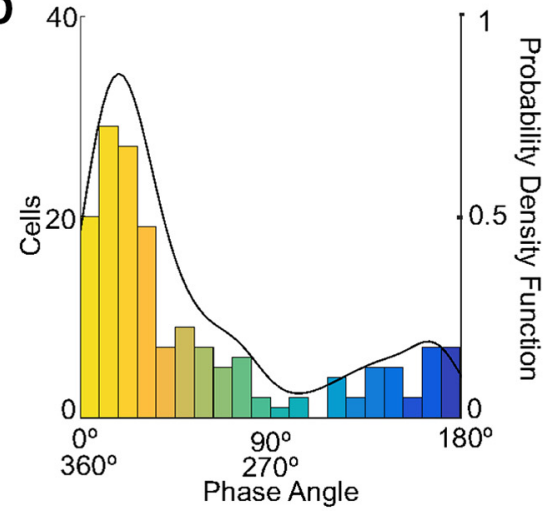

Figure 6. Two functionally defined populations of somatostatin interneurons $A$, Left, (ross-correlation of $\Delta F / F$ versus ball speed during start-triggered events, for positively (red) and negatively (blue) correlated sample cells shown in Figure $5 B, C$. Numbers at peak or nadir of traces indicate cross-correlation lag of $\Delta F / F$ behind speed in seconds. The peak $\Delta F / F$ of the positively correlated cell lags behind speed by $0.8 \mathrm{~s}$. The minimum $\Delta F / F$ of the negatively correlated cell precedes speed by $1 \mathrm{~s}$ in start-triggered events. Right, cross-correlation of stop-triggered events. $\boldsymbol{B}$, Heatmap of the cross-correlation function of $\Delta F / F$ versus ball speed for somatostatin neurons. Cells are sorted in the same order as Figure $5 E$. Time 0 is the zero-lag correlation. Positively correlated cells have activity that peaks close to speed in time (yellow) while negatively correlated cells have activity minima near peak speed (blue). Red and blue arrows mark positively correlated cell shown in $\boldsymbol{B}$, and negatively correlated cell in C, respectively. C, Rose plot of distribution of cells binned by phase angle (by Hilbert transform) of cross-correlation between activity and ball speed. Bars are color-coded by each cell's zero-lag correlation color map of cells in $B$, left. Positively correlated cells have activity that peaks shortly after speed, while negatively correlated cell activity peaks $\sim 180^{\circ}$ out of phase. D, Linear distribution of $\mathrm{C}$ with cells binned from 0 to $180^{\circ}$, and then folding back over from 180 to $360 / 0^{\circ}$. The overlaid trace is the kernel density probability estimate of distribution, which smooths histogram bins.

subset of anticorrelated cells (Fig. 8A-D). We pooled the $r$ values from all parvalbumin interneurons into a single distribution and found that activity in $74.4 \%$ of neurons was correlated with speed, activity in $14.4 \%$ of neurons was anticorrelated with speed, and $11.2 \%$ of neurons had no correlation (Fig. $8 D$; $N=5$ mice, $n=125$ cells; $p<0.05$, Pearson correlation coefficient with Bonferroni-Holm correction for multiple comparisons).

As in the somatostatin population, analysis of start-triggered and stop-triggered activity in the parvalbumin neurons revealed that some neurons increase activity during movement, while activity in a small percentage of cells is inhibited by movement (Fig. $8 E$ ). We quantified this relationship by comparing the fluorescence between immobile and moving periods. Positively correlated cells decreased activity at the transition from moving to immobile (Fig. $8 F ; 0.06 \Delta F / F \pm 0.01$ to $-0.08 \pm .01, p=7.2 \times$ $10^{-9}$, Wilcoxon signed rank) and increased activity at the transition from immobile to moving (Fig. $8 F ;-0.02 \Delta F / F \pm 0.01$ to $0.07 \pm 0.01, n=51$ cells, $\left.p=3.6 \times 10^{-9}\right)$. Conversely, negatively correlated cells increased activity when going from moving to immobile $(-0.03 \Delta F / F \pm 0.03$ to $0.25 \pm 0.05, n=13$ cells, $p=$ $\left.9.7 \times 10^{-4}\right)$ and decreased activity from moving to immobile $\left(0.25 \pm 0.04\right.$ to $\left.0.06 \pm 0.03, p=9.8 \times 10^{-4}\right)$.

Next we examined the temporal relationship between movement and activity by generating a cross-correlation of the speed and activity signals from Figure $8 E$. In positively correlated parvalbumin cells, activity led speed with a short delay (Fig. 8G; $-0.30 \pm 0.18 \mathrm{~s}$ ), in contrast to somatostatin cells where activity slightly lagged speed (Fig. $6 B, C$ ). Activity in negatively correlated parvalbumin cells was much more delayed relative to speed 


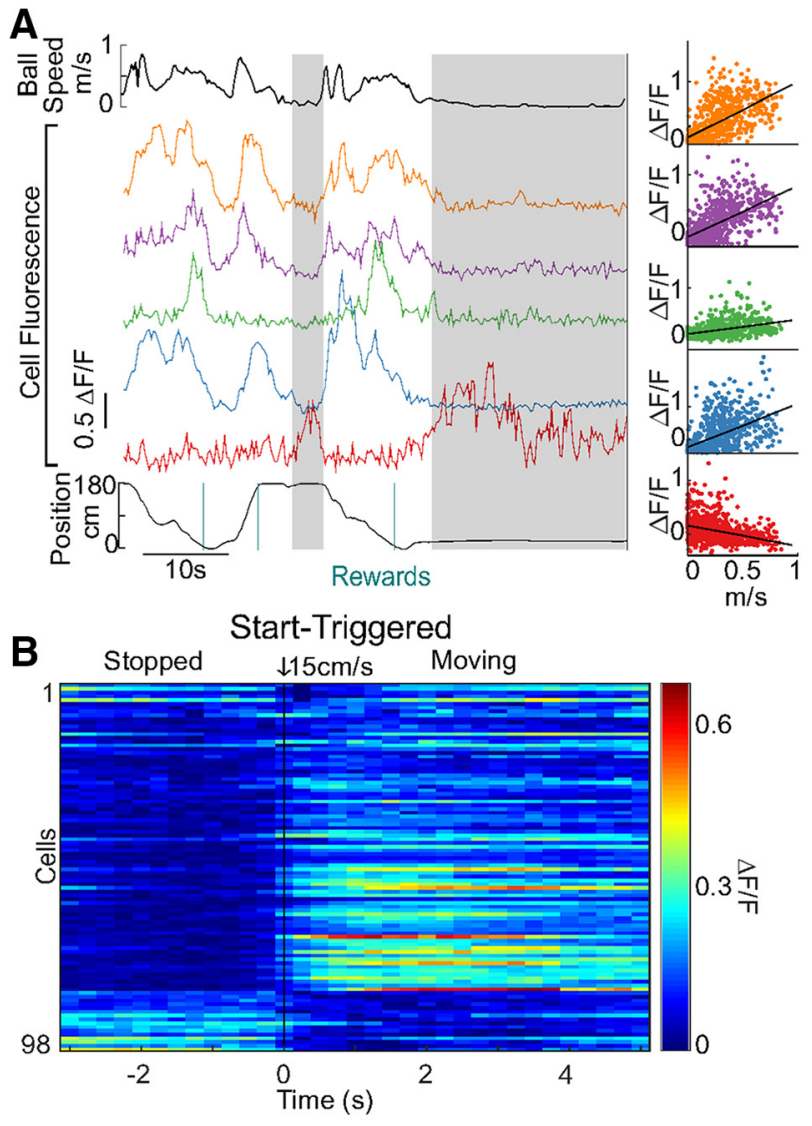

Figure 7. Activity during immobility is not tied to rewards. $\boldsymbol{A}$, Example of activity in neurons during pauses in movement $>4 \mathrm{~s}$ after rewards (shaded boxes). $\boldsymbol{B}$, Locomotion start-triggered events that were $>4$ s from a reward were identified. Rasters of mean $\Delta F / F$ during these events for cells with $>10$ events are shown.

(Fig. 8G; $4.1 \pm 0.35 \mathrm{~s}$ ) and instead the nadir of activity slightly preceded speed (Fig. $8 G ;-0.39 \pm 0.12 \mathrm{~s}$ ).

To test for bimodality in the parvalbumin population, the temporal relationship between activity and speed in start-triggered trials (Fig. 8G, left) was converted to circular statistics by extracting the phase angle of the cross-correlation at zero time lag and plotting the distribution of cells (Fig. $8 H$ ). The resulting distribution shows a statistically significant diametrically bimodal population: one population displays activity that peaks with speed, and a second population is inhibited with speed (phase angle difference of $180^{\circ}$ as tested with Rayleigh $z$ test between the original distribution and the distribution with all angles doubled, $p=1.3 \times$ $\left.10^{-13}\right)$. Together, these data show that two broad classes of genetically specified interneurons-those that express parvalbumin and those that express somatostatin-have populations of cells that are preferentially active during locomotion and immobility.

\section{Anatomy and morphology of cell types}

Are cells with shared functional properties spatially clustered or distributed across the hippocampus? One possible scenario is that positively and negatively correlated cells are enriched in different hippocampal layers. First, to check the gene-expression specificity in these cre-driver lines, we asked whether the anatomical distribution between SO and SP of our recorded neurons (GCaMP-labeled in cre-driver lines) matched the distribution of anti-somatostatin and anti-parvalbumin immunofluorescence in WT mice. The layer-specific position of GCaMP-labeled neurons was determined by examining the individual imaging planes and the separately collected $z$ series of the imaging area. Cells in contact with SP, were scored as SP. For immunofluorescence scoring, we imaged coronal sections and omitted labeled cells in the stratum radiatum (SR) to maintain consistency with GCaMPlabeled distributions, where we did not image into SR. The distribution of GCaMP-labeled neurons and immunofluorescencelabeling was similar (Fig. 9A-C,E; somatostatin: SO, $84.6 \pm 5.5 \%$ GCaMP vs $85.0 \pm 1.6 \%$ immunofluorescence, $p=1$, SP, $15.4 \pm$ $5.5 \%$ GCaMP vs $15.0 \pm 1.6 \%$ immunofluorescence, $p=1$; parvalbumin: SO, $10.1 \pm 3.3 \%$ GCaMP vs $17.5 \pm 2.7 \%$ immunofluorescence, $p=0.14$, SP, $89.9 \pm 3.3 \%$ GCaMP vs $82.5 \pm 2.7 \%$ immunofluorescence, $p=0.08$, with Bonferroni correction; total somatostatin cells: 174 immunolabeled from four mice, 192 GCaMP-labeled from five mice; total parvalbumin cells: 159 immunolabeled from three mice, 125 GCaMP-labeled from five mice).

We then compared the anatomical distribution of positively and negatively correlated cells. There was, within either the parvalbumin and somatostatin populations, no significant difference in the laminar distribution of positively and negatively correlated cells (Fig. 9D, F; somatostatin: SO, $89.5 \pm 4.0 \%$ positive vs $68.5 \pm$ $1.4 \%$ negative, $p=0.28$; SP, $10.5 \pm 4.0 \%$ positive vs $31.5 \pm 1.4 \%$ negative, $p=0.28$; parvalbumin: $\mathrm{SO}, 9.1 \pm 4.5 \%$ positive vs $21.3 \pm 1.1 \%$ negative, $p=1$; SP, $91.0 \pm 4.5 \%$ positive vs $78.8 \pm$ $1.1 \%$ negative, $p=0.06$, with Bonferroni correction). These data demonstrate that positively and negatively correlated cells exhibit approximately the same layer distribution, with somatostatin neurons predominantly in SO and parvalbumin neurons mostly in SP.

Next, we examined the spatial scale of activity correlation in interneuron populations by calculating the temporal activity correlation and spatial distance between pairs of neurons, and then plotting activity correlation as a function of distance. We did this for all somatostatin neurons, then split out positively correlated somatostatin neurons and made pairwise comparisons restricted within this class (Fig. 9G). We were unable to do the same with negatively correlated cells due to the very small number of pairs within each experiment. There was no significant relationship between activity correlation and distance [all somatostatin: Spearman's rank correlation coefficient $(\rho)=0.02, p=0.3, n=$ 2025 pairs, $N=5$ mice; somatostatin positively correlated, $\rho=$ $0.03, p=0.3, n=1110$ pairs]. In contrast, parvalbumin neurons (Fig. $9 H$ ) showed an inverse correlation between activity correlation and distance, when looking at all neurons, as well as within the positively correlated class (all parvalbumin, $\rho=-0.13, p=$ $0.0002, n=996$ pairs, $N=5$ mice; parvalbumin positively correlated, $\rho=-0.14, p=0.0002, n=803$ pairs, with Bonferroni correction). These data show that the closer two parvalbumin neurons are, the more likely they are to have similar activity patterns.

Finally, we examined whether the cell morphology of locomotion-activated neurons was different from that of immobilityactivated neurons. We measured the cross-sectional area and eccentricity of somata from each cell, based on the ROIs used to measure calcium activity. Soma area in negatively correlated somatostatin neurons was significantly smaller than in positively correlated neurons (Fig. 9I, J; $474 \pm 14 \mu \mathrm{m}^{2}$ positive vs $404 \pm 21$ $\mu \mathrm{m}^{2}$ negative, $p=0.025, n=192$ cells, $N=5$ mice). There was no significant difference in eccentricity in somatostatin neurons $(0.77 \pm 0.03$ positive vs $0.75 \pm 0.01$ negative, $p=0.44)$. We also found no significant differences in area and eccentricity between positively and negatively correlated neurons in the parvalbumin populations (area: $453 \pm 11 \mu^{2}$ positive vs $439 \pm 31 \mu \mathrm{m}^{2}$ 

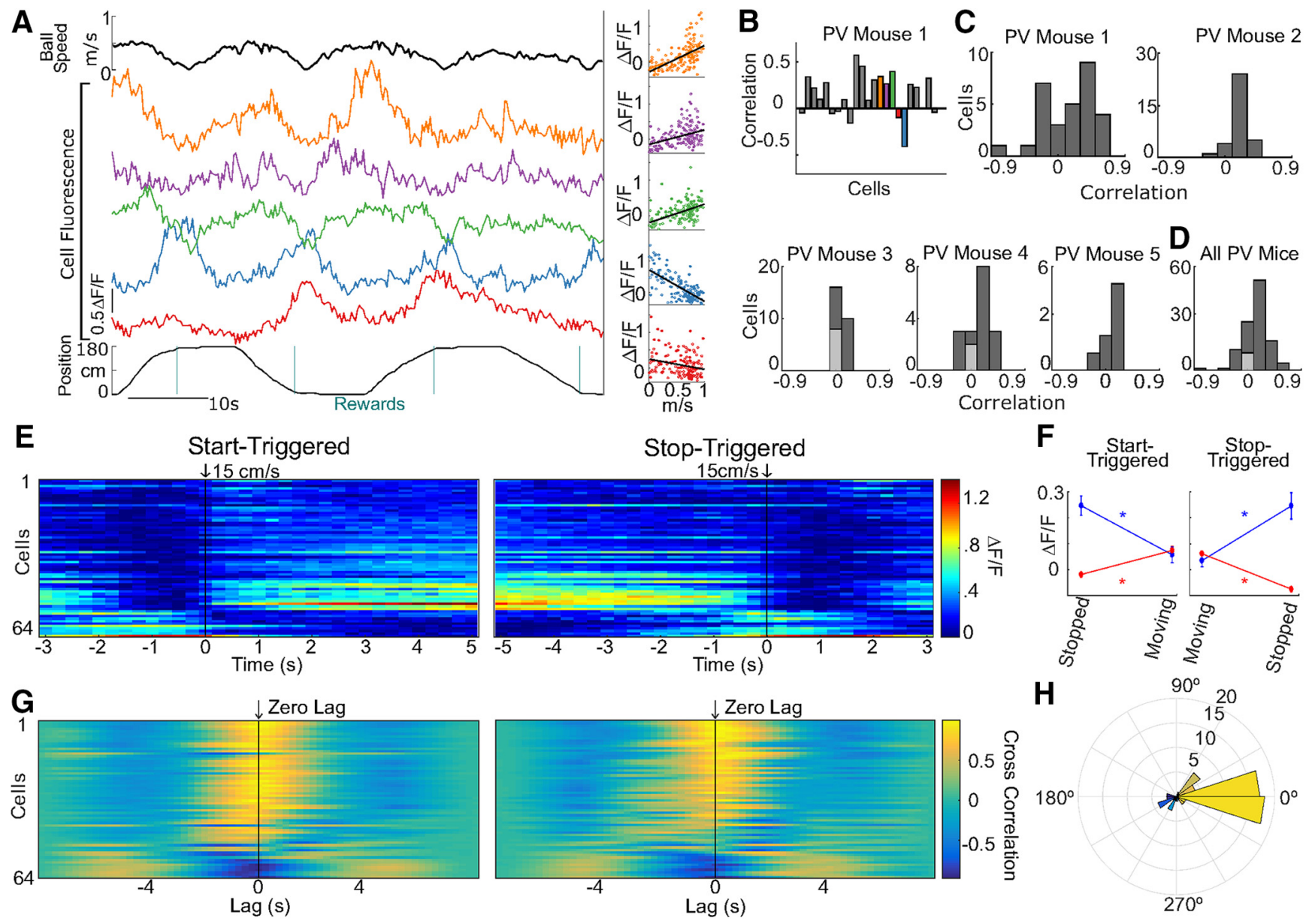

Figure 8. Locomotion-activated and immobility-activated parvalbumin (PV) interneurons. $A, \Delta F / F$ of five sample parvalbumin interneurons plotted with ball speed (top), VR position, and rewards (bottom). Right, Correlation plot of $\Delta F / F$ versus ball speed. Line is linear regression. $B, \Delta F / F$-to-speed correlations for all cells from mouse shown in $A$. Colored bars match individual cells shown in $\boldsymbol{A}$. Solid bar (either dark gray or colored) is a significant relationship, either correlation or anticorrelation. $C$, Distribution of activity-to-speed correlation $r$ values for neurons in individual parvalbumin-cre mice. $\boldsymbol{D}$, Distribution of activity-to-speed correlation $r$ values for all parvalbumin interneurons. $\boldsymbol{E}$, Left, Heatmap of mean of $\Delta F / F$ over time, during start-triggered events for parvalbumin interneurons from experiments with $>10$ start-stop transitions (64 of 125 neurons). Cells with positive activity-to-speed correlation shown at top and negative activity-to-speed correlation at bottom. Within each class, neuron order is sorted from low to high magnitude of correlation or anticorrelation. Right, Heatmap of mean of $\Delta F / F$ over time, during stop-triggered events. Neuron order is the same for start-triggered and stop-triggered heatmaps. $F$, Comparison of mean $\Delta F / F$ ( \pm SEM) between moving and stopped periods for all positively (red) and negatively (blue) correlated cells. ${ }^{*} \leq \leq 9.7 \times 10^{-4}$. G, Left, Heatmap of the cross-correlation function of $\Delta F / F$ versus ball speed in start-triggered events for parvalbumin neurons shown in $E$. Cells are sorted in the same order as $\boldsymbol{E}$. Time 0 is the zero-lag correlation. Positively correlated cells have activity that peaks close to speed in time (yellow), while negatively correlated cells have activity minima near peak speed (blue). Right, Heatmap of the cross-correlation function of $\Delta F / F$ versus ball speed in stop-triggered events. $\boldsymbol{H}$, Rose plot of distribution of cells binned by phase angle (by Hilbert transform) of cross-correlation between activity and ball speed. Bars are color-coded by zero-lag correlation color map of cells in $F$. Positively correlated cells have activity that peaks shortly before speed, while negatively correlated cell activity peaks $\sim 180^{\circ}$ out of phase.

negative, $p=0.71$, eccentricity: $0.75 \pm 0.04$ positive vs $0.84 \pm$ 0.01 negative, $p=0.06, n=125$ cells, $N=5$ mice). These results show that there is no layer preference for positively and negatively correlated neurons (although somatostatin neurons were predominantly in SO and parvalbumin mostly in SP). Furthermore, parvalbumin interneurons show a distance-dependent scaling of activity correlation. Finally, negatively correlated somatostatin interneurons displayed smaller somata than positively correlated interneurons.

\section{Cellular activity-to-speed correlation is stable}

Do neuronal populations that are active or suppressed during locomotion in one environment exhibit stable identities, or are they dynamically reassigned? We examined the stability of these networks in two ways. First, each cell's activity correlation to movement was tracked over time by recording from the same neurons as the animal performed the VR task over $5 \mathrm{~d}$. Indeed, each cell's activity correlation with locomotion was highly stable over this time period (Fig. 10A). To quantify this stability, we plotted each cell's correlation value on different days as a scatter plot and calculated the Pearson correlation value between each day. We found a high degree of correlation of motion response between days (Fig. 10B; day 1 vs day 2, $r=0.83, p=6.5 \times 10^{-65}$; day 1 vs day $3, r=0.75, p=5.4 \times 10^{-47}$; day 1 vs day $4, r=0.71$, $p=6.3 \times 10^{-40}$; day 1 vs day $5, r=0.77, p=5.1 \times 10^{-52}, n=$ 170 cells, $N=5$ mice), indicating that the specification of each cell to a locomotion or immobility activated network is stable over time.

Second, we examined the stability of these functional interneuron networks by transporting animals into a second VR world. In both real and virtual environments, hippocampal place fields remap in different environments (Fig. 2D,E). Is an interneuron's activity correlation to speed similarly environment-dependent or does it remain stable across different environments? After animals were proficient in the behavioral task in one visual environment, they were remapped into a different environment (Fig. 10C). After $5 \mathrm{~d}$ 
of training in the remapping paradigm to allow the animals to become familiar with World 2, we tested network stability by plotting each cell's correlation between activity and locomotion between worlds as a scatter plot. Networks displayed high stability in different environments (Fig. $10 C, D ; r=0.86, p=2.9 \times 10^{-23}, n=75$ cells, $N=3$ mice). These data suggest that a neuron's relationship between activity and movement is a consistent identity. Thus, the hippocampus may contain distinct embedded inhibitory microcircuits encoding locomotor activity versus quiescence.

\section{Discussion}

In this work we applied new experimental approaches to a long-standing question in neuroscience: how is state-dependent activity organized within neural networks? In particular, are hippocampal networks generally responsive during both locomotion and immobility or are there specialized microcircuits in different behavioral states? By imaging hippocampal interneurons in awake, behaving mice, we found two functionally distinct subsets of interneurons with opposite encoding of locomotor state. Specifically, one functional subpopulation is maximally active during locomotion, whereas activity in the second population is anticorrelated with locomotion. Importantly, neither genetic identification nor spatial organization was predictive of a neuron's functional encoding. The stability of these networks over many days and across environments indicates that they likely reflect dedicated circuits that specialize in behavior-dependent processing of hippocampal information.

The existence of two functionally identified classes of interneurons has significant implications for information processing in the hippocampus. In particular, it suggests the possibility that there are "labeled lines" for processing information when an animal is immobile or moving. A network of immobility-activated pyramidal neurons centered in CA2 and extending into CA1 and CA3 has been identified (Kay et al., 2016). One possibility is that these pyramidal neurons preferentially project to immobility-activated CA1 interneurons and drive their activity. Kay et al. identified fast-spiking interneurons whose firing was associated with immobility-activated place cells. However, the proportion of these interneurons that showed a negative correlation between activity and movement was low, suggesting no particular enhanced connectivity between immobility-activated place cells and immobility-activated in-
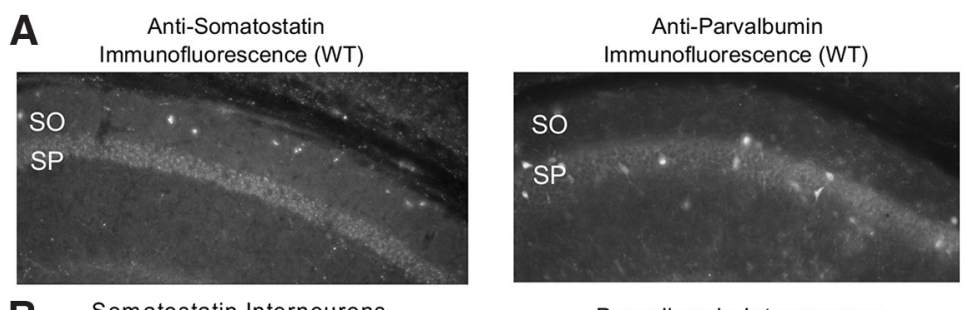

B Somatostatin Interneurons

(SST-cre +/-;

AAV1.Flex.Syn.GCaMP6f)
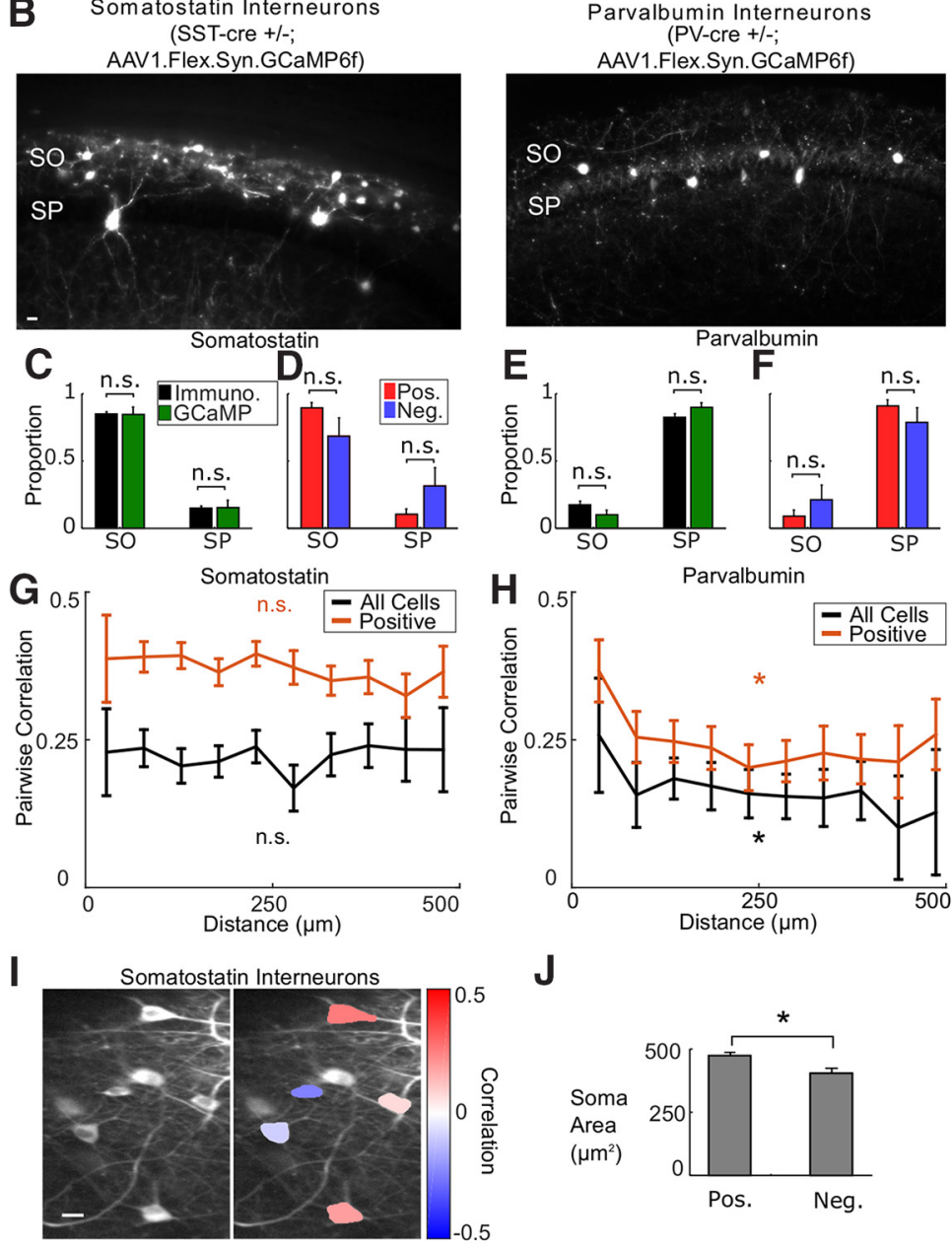

J

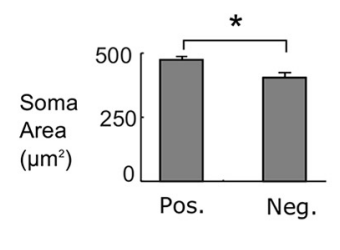

Figure 9. Anatomical distribution and somatic morphology of positively and negatively correlated neurons. A. Left, Antisomatostatin immunofluorescence in coronal section from WT mouse. Right, Anti-parvalbumin immunofluorescence in coronal section from WT mouse. $\boldsymbol{B}$, Left, GCaMP-labeled neurons from somatostatin-cre mouse (coronal section in fixed tissue). Note that SP is dark, indicating the lack of GCaMP-labeled axons targeting cell somata. Right, GCaMP-labeled neurons from parvalbumin-cre mouse. Scale bar, $40 \mu \mathrm{m}$. C, Distribution of cell bodies across cell layers comparing anti-somatostatin immunofluorescence (black) to GCaMP-labeled cells (green). For comparisons in $\boldsymbol{C}-\boldsymbol{F}$, distributions of GCaMP-labeled cells were taken directly from imaging datasets, not post hoc fixed tissue. Cell location was scored by examining imaging planes to see whether cells were in contact with SP, along with detailed $z$ series of imaged cells taken in vivo ( $z$ steps of $3 \mu \mathrm{m}$ through the imaged areas). Differences in $\boldsymbol{C}-\boldsymbol{F}$ were tested by Wilcoxon rank sum, with Bonferroni correction. $\boldsymbol{D}$, Distribution of cell bodies in cell layers from GCaMP-labeled cells in somatostatin-cre animals comparing positively correlated cells (red) to negatively correlated cells (blue). $\boldsymbol{E}$, Distribution of cell bodies across cell layers comparing anti-parvalbumin immunofluorescence (black) to GCaMP-labeled cells (green). $\boldsymbol{F}$, Distribution of cell bodies across cell layers from GCaMP-labeled cells in parvalbumin-cre animals comparing positively correlated cells (red) to negatively correlated cells (blue). G, Plot of mean activity correlation versus distance for pairs of somatostatin neurons, either all possible pairs (All, black) or pairs restricted to the positively correlated population (Positive, red). There was no significant relationship between activity correlation and distance, tested with Spearman's rank correlation, for either all pairs of somatostatin neurons, or just the positively correlated population. $\boldsymbol{H}$, Plot of mean activity correlation versus distance for pairs of parvalbumin neurons, either all possible pairs (All, black) or pairs restricted to the positively correlated population (Positive, red). There was a significant relationship between activity correlation and distance, with closer cell pairs having more similar activity than distant pairs, for all pairs of parvalbumin neurons and for just the positively correlated population. ${ }^{*} p=0.0002$. I, Left, Imaging plane of somatostatin interneurons. Right, Somatic ROls color-coded by activity correlation to speed, with positive correlations in red and negative correlations in blue. ROIs shown here are used to calculate morphology comparisons in J.J, Comparison of cross-sectional area of somatostatin somata between positively correlated and negatively correlated neurons. Areas of somata of negatively correlated somatostatin interneurons were significantly smaller. ${ }^{*} p=0.025$. All error bars are \pm SEM. 
A
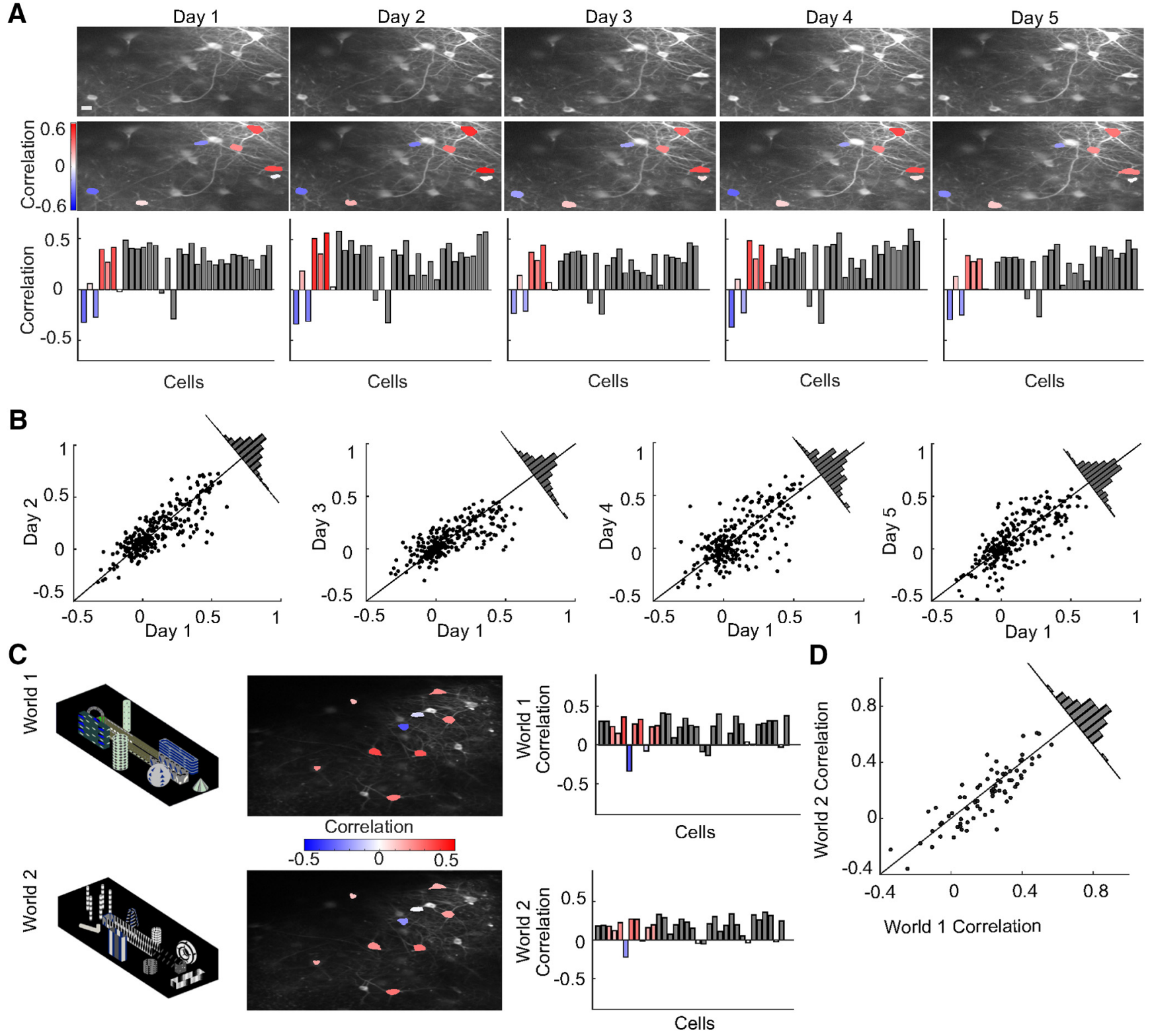

Figure 10. Cellular correlation between activity and locomotion is stable. A, Top, Imaging planes of same somatostatin cells imaged over 5 consecutive days. Scale bar, $20 \mu \mathrm{m}$. Middle, Somata of cells above, color-coded by activity correlation to ball speed over $5 \mathrm{~d}$. Red is positive correlation; blue is negative. Bottom, $\Delta F / F$ correlation to speed for all cells in sample experiment, over $5 \mathrm{~d}$. Cell order is the same across all days. $B$, Scatter plot of $\Delta F / F$ correlation to speed for all cells comparing day 1 to all other days. Inset, Histogram shows distribution of differences between each cell's correlation value between days. Bin size, 0.05 . C, Left, Schematics of World 1 and World 2. Middle, Somata of cells color-coded by activity correlation to ball speed in World 1 and World 2 . Red is positive correlation, blue is negative. Scale bar, $20 \mu \mathrm{m}$. Right, $\Delta F / F$ correlation to speed for all cells in sample experiment, in World 1 and World 2. Cell order is the same across worlds. D, Scatter plot of $\Delta F / F$ correlation to speed for all cells comparing World 1 to World 2. Inset, Histogram shows distribution of differences between each cell's correlation value between worlds. Bin size, 0.05 .

terneurons. Thus, it is possible that other sources may drive the immobility-activated interneurons identified here. Finally, caution should be used when comparing results across experimental paradigms, from tetrode recording in freely moving rats to calcium imaging in head-fixed mice. In particular, the lack of vestibular input (Aronov and Tank, 2014) and nonlinear integration of calcium signals by GCaMP (Chen et al., 2013) could potentially affect the observed relationship between activity and movement.

Another possible local circuit mechanism for generating anticorrelated activity is reciprocal inhibition between the two populations of interneurons. There are inhibitory connections across classes in the hippocampus, both in the direction of parvalbumin interneuron to somatostatin interneuron (Losonczy et al., 2010; Lovett-Barron et al., 2012) and in the direction of somatostatin interneuron to parvalbumin interneuron (Leão et al., 2012; Fuhrmann et al., 2015). Another disinhibition-based local circuit mechanism for generating an immobility-activated population of interneurons is the action of a third class of interneurons, those that express VIP. This class specializes in targeting other interneuron types (Acsády et al., 1996; Tyan et al., 2014; Karnani et al., 2016). This population, if driven by running signals, could inhibit targeted populations during locomotion, and promote activity during immobile periods through disinhibition. There is significant evidence from sensory systems that VIP-expressing interneurons control other interneuron types, and downstream pyramidal neuron activity, in a task-specific manner (Lee et al., 2013; Pi et al., 2013; Fu et al., 2014; Kuchibhotla et al., 2017).

The source of movement and immobility information can also originate from outside the hippocampus. One critical source 
for speed information to the hippocampus is the MS, which is required for running-related $\theta$ oscillations in the hippocampus (Winson, 1978). The MS sends three types of afferent to the hippocampus: glutamatergic, GABAergic, and cholinergic (Frotscher and Léránth, 1985; Freund and Antal, 1988). Glutamatergic input from the MS targets somatostatin-expressing oriens lacunosummoleculare interneurons and drives speed-correlated firing of those interneurons (Fuhrmann et al., 2015). GABAergic input from the MS exclusively targets hippocampal interneurons (Freund and Antal, 1988; Unal et al., 2015) and this connection, which is at least partly active during locomotion (Kaifosh et al., 2013), could serve to inhibit immobility-active neurons during movement. Indeed, this scenario with opposite regulation of activity in two populations of interneuron activity driven by opposing signals from the MS has been hypothesized (Colom and Bland, 1987; Mizumori et al., 1990). Finally, acetylcholine release from MS afferents increases with locomotion and has divergent effects on interneurons, exciting some and inhibiting others (McQuiston and Madison, 1999a,b; Lawrence et al., 2006; Leão et al., 2012; Chittajallu et al., 2013). None of the mechanisms discussed are mutually exclusive; in fact, it is likely that multiple-circuit and neuromodulatory systems are engaged to robustly enforce statedependent network changes on multiple timescales. We note that whatever mechanisms generate these two classes of interneuron require considerable synaptic or cellular specificity. It remains to be seen whether the downstream targets of these interneurons are targeted so precisely.

Our finding of immobility-activated interneurons confirms and expands on earlier work looking for behavioral correlates of hippocampal units using in vivo electrode recordings in freely moving rats. In these studies, occasional "anti- $\theta$ " or $\theta$-off units were identified that increased firing when the animals stopped moving. Since such units were infrequently seen, little was known about them other than they were putatively identified as interneurons (Fox and Ranck, 1975; Buzsáki et al., 1983; Colom and Bland, 1987; Mizumori et al., 1990; Csicsvari et al., 1999). Although we cannot directly confirm that the interneurons we have identified are $\theta$-off units, they share the same functional properties. Through genetic identification, we confirm that these neurons are interneurons and, furthermore, immobility-activated neurons exist in multiple genetically defined interneuronal classes. The fact that this network draws from multiple classes of interneurons with distinct postsynaptic targets suggests a comprehensive circuit for regulating activity during immobile periods. The morphological distinction of smaller somata in immobility-activated somatostatin neurons hints that these functionally defined cell types may correspond to cell classifications based on anatomy or gene expression, although further studies are required to strongly link function to anatomy.

What could be the functional role of these immobilityactivated interneurons? One possibility is that they have a causative role in switching the network from $\theta$ oscillations during movement to large irregular activity during stops, as suggested by studies in urethane-anesthetized rats showing that $\theta$-off units (putative immobility-activated cells) were the first type of unit recruited during LFP transitions (Bland et al., 1999). In addition to possible roles in switching network state, these neurons likely contribute to regulating pyramidal networks. During immobility, there are two well characterized network activities. First are the aforementioned hippocampal pyramidal neurons that code for place during stops. Second are SWRs where firing sequences of pyramidal neurons are reactivated. It is unclear whether these immobility-activated interneurons regulate SWRs since it is un- known whether, or when, SWRs occur during our VR task (although SWRs are associated with consummatory behaviors, such as licking). However, in freely moving animals, SWRs are fast events (100-200 ms) and occupy $<10 \%$ of time during immobile periods (Kay et al., 2016), whereas the activity of immobilityactivated interneurons appears tonic (we note that the temporal resolution of calcium imaging makes it difficult to distinguish tonic activity from periodic phasic activation). Simultaneous imaging and LFP recording may clarify the relationship between immobility-activated interneurons and SWRs. Finally, it is also possible that these interneurons regulate other unknown network activities during immobility.

\section{References}

Acsády L, GörcsTJ, Freund TF (1996) Different populations of vasoactive intestinal polypeptide-immunoreactive interneurons are specialized to control pyramidal cells or interneurons in the hippocampus. Neuroscience 73:317-334. CrossRef Medline

Aghajan ZM, Acharya L, Moore JJ, Cushman JD, Vuong C, Mehta MR (2015) Impaired spatial selectivity and intact phase precession in twodimensional virtual reality. Nat Neurosci 18:121-128. CrossRef Medline

Ahmed OJ, Mehta MR (2012) Running speed alters the frequency of hippocampal gamma oscillations. J Neurosci 32:7373-7383. CrossRef Medline

Aronov D, Tank DW (2014) Engagement of neural circuits underlying 2D spatial navigation in a rodent virtual reality system. Neuron 84:442-456. CrossRef Medline

Bargmann CI, Marder E (2013) From the connectome to brain function. Nat Methods 10:483-490. CrossRef Medline

Berens P (2009) CircStat: A MATLAB Toolbox for Circular Statistics. J Stat Software 31:21. CrossRef

Bland BH, Oddie SD, Colom LV (1999) Mechanisms of neural synchrony in the septohippocampal pathways underlying hippocampal theta generation. J Neurosci 19:3223-3237. Medline

Buzsáki G (2015) Hippocampal sharp wave-ripple: a cognitive biomarker for episodic memory and planning. Hippocampus 25:1073-1188. CrossRef Medline

Buzsáki G, Leung LW, Vanderwolf CH (1983) Cellular bases of hippocampal EEG in the behaving rat. Brain Res 287:139-171. CrossRef Medline

Chen TW, Wardill TJ, Sun Y, Pulver SR, Renninger SL, Baohan A, Schreiter ER, Kerr RA, Orger MB, Jayaraman V, Looger LL, Svoboda K, Kim DS (2013) Ultrasensitive fluorescent proteins for imaging neuronal activity. Nature 499:295-300. CrossRef Medline

Chittajallu R, Craig MT, McFarland A, Yuan X, Gerfen S, Tricoire L, Erkkila B, Barron SC, Lopez CM, Liang BJ, Jeffries BW, Pelkey KA, McBain CJ (2013) Dual origins of functionally distinct O-LM interneurons revealed by differential 5-HT3AR expression. Nat Neurosci 16:1598-1607. CrossRef Medline

Cohen JD, Bolstad M, Lee AK (2017) Experience-dependent shaping of hippocampal CA1 intracellular activity in novel and familiar environments. eLife 6:e23040. CrossRef Medline

Colgin LL (2016) Rhythms of the hippocampal network. Nat Rev Neurosci 17:239-249. CrossRef Medline

Colom LV, Bland BH (1987) State-dependent spike train dynamics of hippocampal formation neurons: evidence for theta-on and theta-off cells. Brain Res 422:277-286. CrossRef Medline

Courtin J, Chaudun F, Rozeske RR, Karalis N, Gonzalez-Campo C, Wurtz H, Abdi A, Baufreton J, Bienvenu TC, Herry C (2014) Prefrontal parvalbumin interneurons shape neuronal activity to drive fear expression. Nature 505:92-96. Medline

Csicsvari J, Hirase H, Czurkó A, Mamiya A, Buzsáki G (1999) Oscillatory coupling of hippocampal pyramidal cells and interneurons in the behaving rat. J Neurosci 19:274-287. Medline

Dana H, Mohar B, Sun Y, Narayan S, Gordus A, Hasseman JP, Tsegaye G, Holt GT, Hu A, Walpita D, Patel R, Macklin JJ, Bargmann CI, Ahrens MB, Schreiter ER, Jayaraman V, Looger LL, Svoboda K, Kim DS (2016) Sensitive red protein calcium indicators for imaging neural activity. eLife 5:e12727. CrossRef Medline

Dombeck DA, Graziano MS, Tank DW (2009) Functional clustering of neurons in motor cortex determined by cellular resolution imaging in awake behaving mice. J Neurosci 29:13751-13760. CrossRef Medline

Dombeck DA, Harvey CD, Tian L, Looger LL, Tank DW (2010) Functional 
imaging of hippocampal place cells at cellular resolution during virtual navigation. Nat Neurosci 13:1433-1440. CrossRef Medline

Ego-Stengel V, Wilson MA (2007) Spatial selectivity and theta phase precession in CA1 interneurons. Hippocampus 17:161-174. CrossRef Medline

Ellender TJ, Nissen W, Colgin LL, Mann EO, Paulsen O (2010) Priming of hippocampal population bursts by individual perisomatic-targeting interneurons. J Neurosci 30:5979-5991. CrossRef Medline

Foster DJ, Wilson MA (2006) Reverse replay of behavioural sequences in hippocampal place cells during the awake state. Nature 440:680-683. CrossRef Medline

Fox SE, Ranck JB Jr (1975) Localization and anatomical identification of theta and complex spike cells in dorsal hippocampal formation of rats. Exp Neurol 49:299-313. CrossRef Medline

Freund TF, Antal M (1988) GABA-containing neurons in the septum control inhibitory interneurons in the hippocampus. Nature 336:170-173. CrossRef Medline

Frotscher M, Léránth C (1985) Cholinergic innervation of the rat hippocampus as revealed by choline acetyltransferase immunocytochemistry: a combined light and electron microscopic study. J Comp Neurol 239:237-246. CrossRef Medline

Fu Y, Tucciarone JM, Espinosa JS, Sheng N, Darcy DP, Nicoll RA, Huang ZJ, Stryker MP (2014) A cortical circuit for gain control by behavioral state. Cell 156:1139-1152. CrossRef Medline

Fuhrmann F, Justus D, Sosulina L, Kaneko H, Beutel T, Friedrichs D, Schoch S, Schwarz MK, Fuhrmann M, Remy S (2015) Locomotion, theta oscillations, and the speed-correlated firing of hippocampal neurons are controlled by a medial septal glutamatergic circuit. Neuron 86:1253-1264. CrossRef Medline

Gentet LJ, Kremer Y, Taniguchi H, Huang ZJ, Staiger JF, Petersen CC (2012) Unique functional properties of somatostatin-expressing GABAergic neurons in mouse barrel cortex. Nat Neurosci 15:607-612. CrossRef Medline

Grewe BF, Voigt FF, van't Hoff M, Helmchen F (2011) Fast two-layer twophoton imaging of neuronal cell populations using an electrically tunable lens. Biomed Opt Express 2:2035-2046. CrossRef Medline

Gu L, Kleiber S, Schmid L, Nebeling F, Chamoun M, Steffen J, Wagner J, Fuhrmann M (2014) Long-term in vivo imaging of dendritic spines in the hippocampus reveals structural plasticity. J Neurosci 34:1394813953. CrossRef Medline

Hinman JR, Brandon MP, Climer JR, Chapman GW, Hasselmo ME (2016) Multiple running speed signals in medial entorhinal cortex. Neuron 91: 666-679. CrossRef Medline

Jung MW, Wiener SI, McNaughton BL (1994) Comparison of spatial firing characteristics of units in dorsal and ventral hippocampus of the rat. J Neurosci 14:7347-7356. Medline

Kaifosh P, Lovett-Barron M, Turi GF, Reardon TR, Losonczy A (2013) Septo-hippocampal GABAergic signaling across multiple modalities in awake mice. Nat Neurosci 16:1182-1184. CrossRef Medline

Karlsson MP, Frank LM (2009) Awake replay of remote experiences in the hippocampus. Nat Neurosci 12:913-918. CrossRef Medline

Karnani MM, Jackson J, Ayzenshtat I, Hamzehei Sichani A, Manoocheri K, Kim S, Yuste R (2016) Opening holes in the blanket of inhibition: localized lateral disinhibition by VIP interneurons. J Neurosci 36:3471-3480. CrossRef Medline

Katona L, Lapray D, Viney TJ, Oulhaj A, Borhegyi Z, Micklem BR, Klausberger T, Somogyi P (2014) Sleep and movement differentiates actions of two types of somatostatin-expressing GABAergic interneuron in rat hippocampus. Neuron 82:872-886. CrossRef Medline

Kay K, Sosa M, Chung JE, Karlsson MP, Larkin MC, Frank LM (2016) A hippocampal network for spatial coding during immobility and sleep. Nature 531:185-190. CrossRef Medline

Kemere C, Carr MF, Karlsson MP, Frank LM (2013) Rapid and continuous modulation of hippocampal network state during exploration of new places. PLoS One 8:e73114. CrossRef Medline

Kropff E, Carmichael JE, Moser MB, Moser EI (2015) Speed cells in the medial entorhinal cortex. Nature 523:419-424. CrossRef Medline

Kuchibhotla KV, Gill JV, Lindsay GW, Papadoyannis ES, Field RE, Sten TA, Miller KD, Froemke RC (2017) Parallel processing by cortical inhibition enables context-dependent behavior. Nat Neurosci 20:62-71. Medline

Lawrence JJ, Statland JM, Grinspan ZM, McBain CJ (2006) Cell typespecific dependence of muscarinic signalling in mouse hippocampal stratum oriens interneurones 10.1113/jphysiol.2005.100875. J Physiol 570:595-610. CrossRef Medline

Leão RN, Mikulovic S, LeãoKE, Munguba H, Gezelius H, Enjin A, Patra K, Eriksson A, Loew LM, Tort AB, Kullander K (2012) OLM interneurons differentially modulate CA3 and entorhinal inputs to hippocampal CA1 neurons. Nat Neurosci 15:1524-1530. CrossRef Medline

Lee S, Kruglikov I, Huang ZJ, Fishell G, Rudy B (2013) A disinhibitory circuit mediates motor integration in the somatosensory cortex. Nat Neurosci 16:1662-1670. CrossRef Medline

Letzkus JJ, Wolff SB, Meyer EM, Tovote P, Courtin J, Herry C, Lüthi A (2011) A disinhibitory microcircuit for associative fear learning in the auditory cortex. Nature 480:331-335. CrossRef Medline

Leutgeb S, Leutgeb JK, Treves A, Moser MB, Moser EI (2004) Distinct ensemble codes in hippocampal areas CA3 and CA1. Science 305:12951298. CrossRef Medline

Losonczy A, Zemelman BV, Vaziri A, Magee JC (2010) Network mechanisms of theta related neuronal activity in hippocampal CA1 pyramidal neurons. Nat Neurosci 13:967-972. CrossRef Medline

Lovett-Barron M, Turi GF, Kaifosh P, Lee PH, Bolze F, Sun XH, Nicoud JF, Zemelman BV, Sternson SM, Losonczy A (2012) Regulation of neuronal input transformations by tunable dendritic inhibition. Nat Neurosci 15: 423-430, S1-S3. CrossRef Medline

Lovett-Barron M, Kaifosh P, Kheirbek MA, Danielson N, Zaremba JD, Reardon TR, Turi GF, Hen R, Zemelman BV, Losonczy A (2014) Dendritic inhibition in the hippocampus supports fear learning. Science 343:857863. CrossRef Medline

Markus EJ, Barnes CA, McNaughton BL, Gladden VL, Skaggs WE (1994) Spatial information content and reliability of hippocampal CA1 neurons: effects of visual input. Hippocampus 4:410-421. CrossRef Medline

McGinley MJ, David SV, McCormick DA (2015) Cortical membrane potential signature of optimal states for sensory signal detection. Neuron 87:179-192. CrossRef Medline

McNaughton BL, Barnes CA, O’Keefe J (1983) The contributions of position, direction, and velocity to single unit activity in the hippocampus of freely-moving rats. Exp Brain Res 52:41-49. Medline

McQuiston AR, Madison DV (1999a) Nicotinic receptor activation excites distinct subtypes of interneurons in the rat hippocampus. J Neurosci 19:2887-2896. Medline

McQuiston AR, Madison DV (1999b) Muscarinic receptor activity has multiple effects on the resting membrane potentials of CA1 hippocampal interneurons. J Neurosci 19:5693-5702. Medline

Mizumori SJ, Barnes CA, McNaughton BL (1990) Behavioral correlates of theta-on and theta-off cells recorded from hippocampal formation of mature young and aged rats. Exp Brain Res 80:365-373. Medline

Morrison DJ, Rashid AJ, Yiu AP, Yan C, Frankland PW, Josselyn SA (2016) Parvalbumin interneurons constrain the size of the lateral amygdala engram. Neurobiol Learn Mem 135:91-99. CrossRef Medline

Mukamel EA, Nimmerjahn A, Schnitzer MJ (2009) Automated analysis of cellular signals from large-scale calcium imaging data. Neuron 63:747760. CrossRef Medline

Muller RU, Kubie JL (1987) The effects of changes in the environment on the spatial firing of hippocampal complex-spike cells. J Neurosci 7:19511961. Medline

Niell CM, Stryker MP (2010) Modulation of visual responses by behavioral state in mouse visual cortex. Neuron 65:472-479. CrossRef Medline

O'Keefe J, Dostrovsky J (1971) The hippocampus as a spatial map. Preliminary evidence from unit activity in the freely-moving rat. Brain Res 34: 171-175. CrossRef Medline

Pakan JM, Lowe SC, Dylda E, Keemink SW, Currie SP, Coutts CA, Rochefort NL (2016) Behavioral-state modulation of inhibition is contextdependent and cell type specific in mouse visual cortex. eLife 5:e14985. CrossRef Medline

Peron SP, Freeman J, Iyer V, Guo C, Svoboda K (2015) A cellular resolution map of barrel cortex activity during tactile behavior. Neuron 86:783-799. CrossRef Medline

Pi HJ, Hangya B, Kvitsiani D, Sanders JI, Huang ZJ, Kepecs A (2013) Cortical interneurons that specialize in disinhibitory control. Nature 503: 521-524. CrossRef Medline

Polack PO, Friedman J, Golshani P (2013) Cellular mechanisms of brain state-dependent gain modulation in visual cortex. Nat Neurosci 16:13311339. CrossRef Medline

Ranck JB Jr (1973) Studies on single neurons in dorsal hippocampal forma- 
tion and septum in unrestrained rats. I. Behavioral correlates and firing repertoires. Exp Neurol 41:462-531. CrossRef Medline

Reimer J, Froudarakis E, Cadwell CR, Yatsenko D, Denfield GH, Tolias AS (2014) Pupil fluctuations track fast switching of cortical states during quiet wakefulness. Neuron 84:355-362. CrossRef Medline

Royer S, Zemelman BV, Losonczy A, Kim J, Chance F, Magee JC, Buzsáki G (2012) Control of timing, rate and bursts of hippocampal place cells by dendritic and somatic inhibition. Nat Neurosci 15:769-775. CrossRef Medline

Schneider DM, Nelson A, Mooney R (2014) A synaptic and circuit basis for corollary discharge in the auditory cortex. Nature 513:189-194. CrossRef Medline

Stefanelli T, Bertollini C, Lüscher C, Muller D, Mendez P (2016) Hippocampal somatostatin interneurons control the size of neuronal memory ensembles. Neuron 89:1074-1085. CrossRef Medline

Taniguchi H, He M, Wu P, Kim S, Paik R, Sugino K, Kvitsiani D, Fu Y, Lu J, Lin Y, Miyoshi G, Shima Y, Fishell G, Nelson SB, Huang ZJ (2011) A resource of cre driver lines for genetic targeting of GABAergic neurons in cerebral cortex. Neuron 71:995-1013. CrossRef Medline

Tyan L, Chamberland S, Magnin E, Camiré O, Francavilla R, David LS, Deisseroth K, Topolnik L (2014) Dendritic inhibition provided by interneuron-specific cells controls the firing rate and timing of the hippocampal feedback inhibitory circuitry. J Neurosci 34:4534-4547. CrossRef Medline
Unal G, Joshi A, Viney TJ, Kis V, Somogyi P (2015) Synaptic targets of medial septal projections in the hippocampus and extrahippocampal cortices of the mouse. J Neurosci 35:15812-15826. CrossRef Medline

Vanderwolf CH (1969) Hippocampal electrical activity and voluntary movement in the rat. Electroencephalogr Clin Neurophysiol 26:407-418. CrossRef Medline

Varga C, Golshani P, Soltesz I (2012) Frequency-invariant temporal ordering of interneuronal discharges during hippocampal oscillations in awake mice. Proc Natl Acad Sci U S A 109:E2726-E2734. CrossRef Medline

Vinck M, Batista-Brito R, Knoblich U, Cardin JA (2015) Arousal and locomotion make distinct contributions to cortical activity patterns and visual encoding. Neuron 86:740-754. CrossRef Medline

Wilson MA, McNaughton BL (1993) Dynamics of the hippocampal ensemble code for space. Science 261:1055-1058. CrossRef Medline

Wilson MA, McNaughton BL (1994) Reactivation of hippocampal ensemble memories during sleep. Science 265:676-679. CrossRef Medline

Winson J (1978) Loss of hippocampal theta rhythm results in spatial memory deficit in the rat. Science 201:160-163. CrossRef Medline

Zar JH (2007) Biostatistical analysis, 4th edition: Upper Saddle River, NJ: Prentice Hall.

Zheng C, Bieri KW, Trettel SG, Colgin LL (2015) The relationship between gamma frequency and running speed differs for slow and fast gamma rhythms in freely behaving rats. Hippocampus 25:924-938. CrossRef Medline 